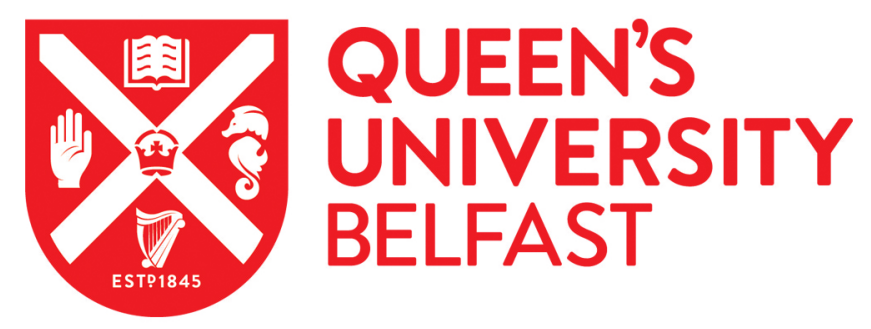

\title{
Development and characterisation of novel poly (vinyl alcohol)/poly (vinyl pyrrolidone)-based hydrogel-forming microneedle arrays for enhanced and sustained transdermal delivery of methotrexate
}

Tekko, I. A., Chen, G., Domínguez-Robles, J., Thakur], R. R. S., Hamdan, I. M. N., Vora, L., Larrañeta, E., McElnay, J. C., McCarthy, H. O., Rooney, M., \& Donnelly, R. F. (2020). Development and characterisation of novel poly (vinyl alcohol)/poly (vinyl pyrrolidone)-based hydrogel-forming microneedle arrays for enhanced and sustained transdermal delivery of methotrexate. International Journal of Pharmaceutics, [119580]. https://doi.org/10.1016/j.ijpharm.2020.119580

Published in:

International Journal of Pharmaceutics

Document Version:

Peer reviewed version

Queen's University Belfast - Research Portal:

Link to publication record in Queen's University Belfast Research Portal

\section{Publisher rights}

(C) 2020 Elsevier B.V.

This manuscript is distributed under a Creative Commons Attribution-NonCommercial-NoDerivs License

(https://creativecommons.org/licenses/by-nc-nd/4.0/), which permits distribution and reproduction for non-commercial purposes, provided the author and source are cited.

\section{General rights}

Copyright for the publications made accessible via the Queen's University Belfast Research Portal is retained by the author(s) and / or other copyright owners and it is a condition of accessing these publications that users recognise and abide by the legal requirements associated with these rights.

\section{Take down policy}

The Research Portal is Queen's institutional repository that provides access to Queen's research output. Every effort has been made to ensure that content in the Research Portal does not infringe any person's rights, or applicable UK laws. If you discover content in the Research Portal that you believe breaches copyright or violates any law, please contact openaccess@qub.ac.uk. 


\section{Journal Pre-proofs}

Development and characterisation of novel poly (vinyl alcohol)/poly (vinyl pyrrolidone)-based hydrogel-forming microneedle arrays for enhanced and sustained transdermal delivery of methotrexate

Ismaiel A. Tekko, Gaoyun Chen, Juan Domínguez-Robles, Raghu Raj Singh Thakur, Iman M.N. Hamdan, Lalitkumar Vora, Eneko Larrañeta, James C. McElnay, Helen O. McCarthy, Madeleine Rooney, Ryan F. Donnelly

PII: S0378-5173(20)30564-0

DOI: https://doi.org/10.1016/j.ijpharm.2020.119580

Reference: IJP 119580

To appear in: $\quad$ International Journal of Pharmaceutics

Received Date: $\quad 14$ May 2020

Revised Date: $\quad 20$ June 2020

Accepted Date: $\quad 22$ June 2020

Please cite this article as: I.A. Tekko, G. Chen, J. Domínguez-Robles, R. Raj Singh Thakur, I.M.N. Hamdan, L. Vora, E. Larrañeta, J.C. McElnay, H.O. McCarthy, M. Rooney, R.F. Donnelly, Development and characterisation of novel poly (vinyl alcohol)/poly (vinyl pyrrolidone)-based hydrogel-forming microneedle arrays for enhanced and sustained transdermal delivery of methotrexate, International Journal of Pharmaceutics (2020), doi: https:// doi.org/10.1016/j.ijpharm.2020.119580

This is a PDF file of an article that has undergone enhancements after acceptance, such as the addition of a cover page and metadata, and formatting for readability, but it is not yet the definitive version of record. This version will undergo additional copyediting, typesetting and review before it is published in its final form, but we are providing this version to give early visibility of the article. Please note that, during the production process, errors may be discovered which could affect the content, and all legal disclaimers that apply to the journal pertain.

(C) 2020 Elsevier B.V. All rights reserved. 
Development and characterisation of novel poly (vinyl alcohol)/poly (vinyl pyrrolidone)based hydrogel-forming microneedle arrays for enhanced and sustained transdermal delivery of methotrexate

Ismaiel A. Tekko a,b, Gaoyun Chen ${ }^{\mathrm{a}}$, Juan Domínguez-Robles ${ }^{\mathrm{a}}$, Raghu Raj Singh Thakur ${ }^{\mathrm{a}}$, Iman M.N. Hamdanc, Lalitkumar Vora ${ }^{\mathrm{a}}$, Eneko Larrañeta ${ }^{\mathrm{a}}$, James C. McElnaya , Helen O. McCarthya, Madeleine Rooney ${ }^{\mathrm{d}^{*}}$ and Ryan F. Donnelly,*

a School of Pharmacy, Medical Biology Centre, Queen's University Belfast, 97 Lisburn Road, Belfast, BT9 7BL, Northern Ireland, United Kingdom

${ }^{b}$ Faculty of Pharmacy, Aleppo University, Aleppo, Syria

${ }^{c}$ Faculty of Pharmacy, Middle East University, Amman 11831, Jordan

${ }^{\mathrm{d}}$ Centre for Experimental Medicine (CEM), School of Medicine, Dentistry and Biomedical Sciences Queen's University Belfast, 97 Lisburn Road, Belfast, BT9 7BL, Northern Ireland, United Kingdom

*Corresponding authors:

- Professor Ryan F. Donnelly

Chair in Pharmaceutical Technology

School of Pharmacy

Queens University Belfast

Medical Biology Centre

97 Lisburn Road, Belfast, BT9 7BL, Northern Ireland, United Kingdom

Tel: +44 (0) 2890972251

Fax: +44 (0) 2890247794

Email: R.Donnelly@qub.ac.uk

- Dr Madeleine Rooney

Clinical Senior Lecturer

Centre for Experimental Medicine

School of Medicine, Dentistry and Biomedical Sciences

97 Lisburn Road, Belfast, BT9 7BL, Northern Ireland, United Kingdom

Tel: +44 (0)289097 2909

Email: M.Rooney@qub.ac.uk 


\section{Abstract}

Methotrexate (MTX) is one of the mainstays of treatment for rheumatoid arthritis (RA) and juvenile idiopathic arthritis (JIA) and it is mainly administered either orally or by subcutaneous (SC) injection, which are not so satisfactory. While orally administered MTX is associated with variable bioavailability and causes gastrointestinal side effects, including nausea and vomiting, SC injection is painful and produces high peak blood levels of MTX. Transdermal delivery presents an attractive alternative administration route. However, MTX passive permeation through the skin is hindered by the skin barrier and MTX physicochemical properties. To address these issues, hydrogel-forming microneedle arrays (HFMN) and a patch-like reservoir loaded with MTX (MTX-RV) were developed and combined to form a minimally invasive patch to deliver MTX transdermally in a sustained manner. HFMN were prepared from an aqueous blend of poly (vinyl alcohol) (PVA) and poly (vinyl pyrrolidone) (PVP) which was crosslinked chemically with citric acid $(\mathrm{CA})$ at $130^{\circ} \mathrm{C}$. MTX-RV was prepared from hydroxypropyl methylcellulose (HPMC) and glycerol. Both the HFMN and MTX-RV were fully characterised and then combined to form an integrated patch, which was evaluated ex vivo and in preclinical studies. The HFMN demonstrated a satisfactory mechanical strength and insertion capability into excised neonatal porcine skin, as well as moderate swelling properties. The MTX-RV incorporated a high dose of MTX $(150.3 \pm 5.3 \mu \mathrm{g} / \mathrm{mg})$ without precipitation. The integrated patch delivered MTX at a steady-state flux of $506.8 \pm 136.9 \mu \mathrm{g} . \mathrm{cm}^{2} / \mathrm{h}$ in an ex vivo setup. Furthermore, in preclinical studies performed in Sprague Dawley rats, MTX appeared in blood after $1 \mathrm{~h}$ from patch application at a concentration of $7.6 \pm 2.0 \mathrm{nM}$. MTX blood level increased gradually to reach its peak, $\mathrm{C}_{\max }=35.1 \pm 5.1 \mathrm{nM}$, at $24 \mathrm{~h}$. Importantly, the HFMN were removed intact from the skin with only mild erythema, despite the cytotoxic nature of MTX. Accordingly, the integrated patch produced in this work represents a promising minimally invasive transdermal drug delivery system that can overcome the skin barrier and deliver MTX in a sustained manner. This may help in minimising or even avoiding the nausea and vomiting, associated with the conventional administration routes.

Keywords: Methotrexate; juvenile idiopathic arthritis; transdermal delivery; hydrogel-forming microneedles; crosslinking; sustained release. 


\section{Introduction}

Methotrexate (MTX) is a folate antagonist with anti-inflammatory properties [1]. It is one of the mainstays of treatment for several immune-mediated inflammatory diseases, including rheumatoid arthritis (RA) and juvenile idiopathic arthritis (JIA) [2,3]. Indeed, MTX is still the only disease-modifying drug used for the treatment of both RA and JIA before the use of expensive biologics, such as adalimumab, infliximab and certolizumab $[1,2,4]$. MTX is used to achieve fast disease control to prevent long-term damaging effects with a dose range of 7.5- $25 \mathrm{mg}$ once weekly [4,5].

The most common and convenient administration route for MTX is the oral route [6]. However, orally administered MTX has a variable intestinal absorption and nonlinear pharmacokinetics, especially at doses higher than $15 \mathrm{mg}$, with significant consequences on drug bioavailability and clinical efficacy [7-10]. Additionally, MTX administered by this route is associated with gastrointestinal (GIT) side effects, such as nausea, vomiting, diarrhoea, abdominal pain, anorexia and stomatitis, that frequently lead to treatment cessation or switching to expensive biologics in more than $50 \%$ of patients $[3,6,11,12]$. To overcome these limitations, several strategies have been investigated, including using folic acid and folinic acid supplements [6,13], dose titration [4] splitting the weekly oral dose into two or more doses given at 12-hour intervals [14], with limited success [4,15]. The most effective approach has been bypassing the GIT by switching the administration route to subcutaneous (SC) injection [9]. Despite its proven advantages in combating the variable intestinal absorption and nonlinear pharmacokinetics and mitigating many of the drug side effects associated with oral administration, SC injection is still associated with nausea and vomiting [4,5], which is likely due to the high peak blood levels of MTX produced by this administration route $[6,15]$. Additionally, SC injection is painful and stressful for patients, especially children, which may affect patient compliance $[5,7,16,17]$. To overcome these limitations, alternative administration route and drug delivery system that avoid the GIT and the high peak blood levels of MTX are highly desirable.

Transdermal delivery route is an attractive alternative administration route as it has several advantages, including (i) avoiding the gastrointestinal tract and the non-linear pharmacokinetics, thus minimising the GIT side effects (ii) evading the inconvenient and painful SC injection, thus improving patient compliance [18]. However, MTX transdermal delivery is hindered by the skin`s strong barrier and MTX physicochemical properties, including being a hydrophilic drug with $\log \mathrm{P}$ of -1.85 and has an ionised structure at the 
physiological $\mathrm{pH}[19,20]$. Many strategies have been investigated to overcome the skin barrier for MTX transdermal permeation, including use of niosomes, liposomes, nanogels, electroporation, sonophoresis, iontophoresis and dissolving microneedles (MN) [18,21]. Despite increased drug permeation, it is still challenging to administer a therapeutically effective dose of MTX using any of these methods [18,21]. Recently, another type of MN, namely solid MN made of silicon and PLGA have been investigated to enhance MTX transdermal delivery [22,23]. However, these solid MN have only been investigated in an in vitro. Thus, the therapeutic effects are yet to be tested in vivo. Additionally, in this approach, a two-step "poke and patch" technique was used, by which MN merely are employed to generate microchannels in the skin before the application of a topical MTX formulation. This approach has shown to be burdensome and error-prone [24]. Moreover, the micropores generated by MN are quickly closed, resulting in reduced drug permeation [24]. Therefore, an alternative drug delivery system which can overcome these limitations is needed.

Hydrogel-forming microneedle arrays (HFMN) are an attractive alternative MN type first described by our Group [25]. In addition to many advantages, such as being minimally invasive, can be self-applied with minimal instructions, self-disabling through in -situ softening, thus no sharp wastes and requiring only one-step application. The HFMN are made from crosslinked polymers and the drug is contained in an attached patch-like drug reservoir. Thus 10s of milligrams of the drug, which is contained in the reservoir, can be delivered can delivered $[25,26]$. Upon insertion, the HFMN rapidly absorb skin interstitial fluid and swell to form continuous and unblockable conduits between the attached drug reservoir and the dermal microcirculation. Thus, considerable amounts of the drug from the attached reservoir can be delivered [25]. Moreover, HFMN can be removed macroscopically intact from the skin; thus, only minimal unmeasurable amounts of polymers are deposited in the skin [26]. This approach has already demonstrated superiority in delivering high doses of many small drug molecules as well as macromolecules, such as metformin and bevacizumab [25,27-29].

Although HFMN have existed for nearly a decade, the number of fabrication materials is still limited, and most HFMN are prepared from Gantrez ${ }^{\circledR}$ AN-139 or Gantrez ${ }^{\circledR}$ S-97 crosslinked with polyethylene glycol (PEG) [24,26]. Despite their usefulness in delivering many drugs, Gantrez ${ }^{\circledR}$-based HFMN`s ability to deliver MTX is reduced due to chemical incompatibility. This is because Gantrez ${ }^{\circledR}$ AN-139 and Gantrez ${ }^{\circledR}$ S-97 are copolymers of methyl vinyl ether and maleic anhydride and methyl vinyl ether and maleic acid, respectively, containing a considerable number of carboxylic acid groups. Upon crosslinking with PEG to 
form the abovementioned HFMN, a high proportion of the negatively charged carboxylic groups remain unreacted [30]. MTX (Figure 1) is a weak bicarboxylic acid with several amino groups in its structure and possess two pKa values; 4.71 and 3.36 [20]. At low pH, MTX aqueous solubility is reduced dramatically and its amino groups become ionised [20]. Thus, upon contact with the hydrated Gantrez ${ }^{\circledR}$-based HFMN, MTX may precipitate and its positively charged amino groups can interact with the negatively charged acid groups from Gantrez ${ }^{\circledR}$, forming a complex, thus reducing its diffusion through the HFMN and delivery to the dermal microcirculation. To overcome this limitation, new HFMN prepared from MTX-compatible polymeric materials is required.

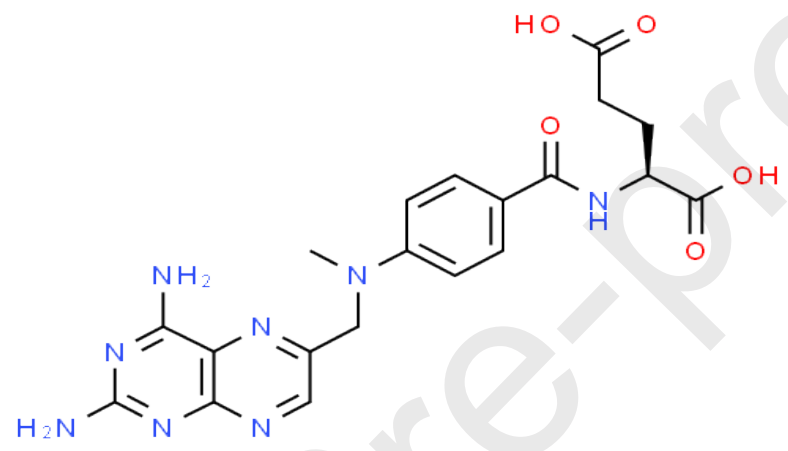

Figure1. The chemical structure of methotrexate.

The present work describes, for the first time, the development of HFMN arrays prepared from biocompatible and chemically MTX-compatible polymeric materials, and MTX-loaded patch-like polymeric reservoir (MTX-RV) that combined to deliver MTX transdermally in a sustained manner.

\section{Materials and Methods}

\subsection{Materials}

Poly (vinyl alcohol) (PVA, Mw = $85-124 \mathrm{kDa}, 87-89 \%$ hydrolysed), poly (ethylene glycol) (PEG 4000, Mw $=400$ and (PEG 10,000, Mw 10,000 Da), poly (vinyl pyrrolidone) K90 (PVP K90, Mw = $360 \mathrm{kDa}$ ) and hydroxypropyl methylcellulose (HPMC) with a viscosity of 40 - 60 centipoise, $2 \%$ in $\mathrm{H}_{2} \mathrm{O}\left(20^{\circ} \mathrm{C}\right)$ were purchased from Sigma-Aldrich (Dorset, UK). Citric acid (CA) was supplied by BDH Laboratory Supplies (Poole, UK). PVP K29-32 (Mw = $58 \mathrm{kDa}$ ) was kindly donated by Ashland (Surrey, UK). Methotrexate disodium (MTX) (99.35\% purity) was purchased from Haihang Industry Co., Ltd (Shandong, China). 
Methotrexate polyglutamates $\left(\mathrm{MTX}^{-} \mathrm{PG}_{1-5}\right)$ standards were purchased from Schircks Laboratories (Jona, Switzerland). Guthrie cards (Schleicher \& Schuell 903) were purchased from Aston Ltd. (Oldham, England). Oasis solid-phase extraction (SPE) cartridges were obtained from Waters (Dublin, Ireland). Glycerine (99.5\% purity) was purchased from VWR International (Lutterworth, England). HPLC grade water was produced using a Millipore Direct-Q ${ }^{\mathrm{TM}} 5$ water purification system from Millipore (Watford, England). All other chemicals and materials were of analytical reagent grade supplied by Sigma-Aldrich (Dorset, UK) and Fisher Scientific (Loughborough, UK).

\subsection{Preparation and characterisation of hydrogel-forming films (HFFs)}

HFMN arrays were fabricated from aqueous polymeric blends containing mainly a biocompatible polymer, PVA and CA (as a crosslinking agent), dried and crosslinked by heating at $130^{\circ} \mathrm{C}$. Prior to preparation of HFMN, the blend compositions and crosslinking time were finalised by fabrication and characterisation of hydrogel-forming films (HFFs), which is a widely used approach $[25,26,31]$. The blend compositions optimisation was achieved by investigating the effect of PVA concentration (F1, F2 and F3), CA concentrations (F4), adding other materials such as glycerol (F5) and PEG 400 and 10,000 (F6 and F7, respectively), and varying concentrations of PVP (F8, F9 and F10) on mechanical and swelling properties, the percentage of gel fraction of the HFFs and their permeability to MTX. The compositions of the aqueous PVA-based polymeric blends are outlined in Table 1.

Table 1. Composition of the aqueous PVA-based polymeric blends used to prepare HFFs.

\begin{tabular}{ccccccccccc}
\hline & \multicolumn{10}{c}{ Formulae (100 g) } \\
\hline $\begin{array}{c}\text { Formulae } \\
\text { code }\end{array}$ & F1 & F2 & F3 & F4 & F5 & F6 & F7 & F8 & F9 & F10 \\
\hline Ingredients & & & \multicolumn{1}{c}{$\mathbf{~ \% ~ w / w ~}$} \\
\hline PVA & 5 & 10 & 15 & 15 & 15 & 15 & 15 & 15 & 15 & 15 \\
CA & 0.5 & 1 & 1.5 & 3 & 1.5 & 1.5 & 1.5 & 1.5 & 1.5 & 1.5 \\
Glycerol & - & - & - & - & 5 & - & - & - & - & - \\
PEG 400 & - & - & - & - & - & 5 & - & - & - & - \\
PEG 10,000 & - & - & - & - & - & - & 5 & - & - & - \\
PVP & - & - & - & - & - & - & & 5 & 7.5 & 10 \\
DI water & 94.5 & 89 & 83.5 & 82 & 78.5 & 78.5 & 78.5 & 78.5 & 76 & 73.5 \\
\hline
\end{tabular}

To prepare the above described polymeric blends, aqueous solutions with varying PVA concentrations were first made using the required weight of PVA. This was achieved by adding PVA to an ice-cold deionised (DI) water and stirring vigorously to ensure complete wetting and prevention of aggregation. The mixture was then heated and maintained at $95 \pm 1^{\circ} \mathrm{C}$ until 
a clear solution was obtained. Upon cooling, pre-determined amounts of the crosslinking agentCA, alone or with either glycerol, PEG 400, PEG 10,000 or varying concentrations of PVP were added. Finally, these polymeric blends were adjusted to their final weight with DI water, homogenised and centrifuged at 3500 RPM for 15 minutes to remove any air bubbles.

To prepare the HFFs, an aliquot (10 g) from each polymeric blend was slowly poured into an in-house made mould consisting of a release liner, with the siliconized side up, secured to a Perspex baseplate with a stainless-steel clamp. Once assembled, the internal dimensions available for casting were $55 \times 55 \mathrm{~mm}^{2}$ and the side wall's height was $4 \mathrm{~mm}$. The mould was placed on a levelled surface, allowing the blend to spread evenly across the area of the mould. The cast blends were dried for $48 \mathrm{~h}$ at room temperature to form films. After drying, the films were cured at $130 \pm 1{ }^{\circ} \mathrm{C}$ for 40 min to induce ester-based crosslinking between PVA and CA to form the insoluble HFFs [32]. The resultant HFFs were then slowly cooled at room temperature and stored in sealed aluminium bags with added silica gel pouches until further use. The optimal crosslinking temperature was identified to be $130^{\circ} \mathrm{C}$ [32]. However, the crosslinking time needed further optimisation. To this end, HFFs, which were prepared from selected polymeric blends (those shown promising properties) were crosslinked at the same temperature for either 120 or $180 \mathrm{~min}$. For non-crosslinked films, after being dried, these were removed and stored in resealable polyethene bags until further use.

\subsubsection{Assessment of the mechanical properties of the HFFs}

The mechanical properties of the HFFs were assessed by measuring their break/bend strength and determining the maximum axial force $\left(F_{\max }\right)$ to break/bend a $(10 \times 10 \mathrm{~mm})$ segment from the HFFs using a TA-XT2 Texture Analyser (Stable Micro Systems, Surrey, UK) operated in compression mode as previously described [33,34]. $\mathrm{F}_{\max }$ was calculated from the resultant force-distance curve. Since the mechanical properties of the HFFs will be affected by their thickness. This was measured using a digital micro-calliper at six different points. All the tests were carried out at room temperature.

\subsubsection{Assessment of the swelling kinetics and percentage of gel fraction of the HFFs}

The swelling properties of the HFFs were investigated by direct immersion of the studied HFFs in DI water at room temperature. Segments $(10 \times 10 \mathrm{~mm})$ of the HFFs were first 
weighed in the dry state $\left(\mathrm{M}_{0}\right)$ and then placed into DI water $(20 \mathrm{ml})$ at room temperature for $24 \mathrm{~h}$. The segments were removed from the water at pre-determined time intervals $(\mathrm{t})$, dried gently with filter paper to eliminate the excess surface water and then weighed again at each time point $\left(\mathrm{M}_{\mathrm{t}}\right)$. The percentage of swelling (\% Swelling) was calculated using Equation 1 [26]. To calculate the percentage of swelling at equilibrium (\%SE), Equation 1 was again employed. In this case, $M_{t}$ was the weight of the HFFs at the time interval $(t=24 h)$ in which HFFs were in a fully swollen state (i.e. swelling at equilibrium).

$$
\% \text { Swelling }=\frac{M_{t}-M_{0}}{M_{0}} x 100 \quad \text { Equation } 1
$$

To evaluate the efficiency of crosslinking reaction to form an insoluble HFMN that can be removed macroscopically intact from the skin, the percentage of gel fraction (\%GF) was determined. \%GF refers to the percentage of the insoluble polymer network formed upon crosslinking $[35,36]$. The $\% \mathrm{GF}$ was determined by collecting the fully hydrated segments of the HFFs from the swelling studies and the excess surface water was gently eliminated with filter paper and then dried in a pre-heated oven at $80 \pm 1{ }^{\circ} \mathrm{C}$ until constant weight values were obtained. This process allows the removal of all the water and the unreacted leachable materials/polymers from the films leaving only the insoluble polymer network behind. \%GF was calculated using Equation 2 [35].

$$
\% G F=\frac{\mathrm{M}_{\mathrm{d}}}{\mathrm{M}_{0}} \times 100 \quad \text { Equation } 2
$$

Where $\mathrm{M}_{\mathrm{d}}$ is the weight of dehydrated HFFs that dried in an oven at $80 \pm 1{ }^{\circ} \mathrm{C}$, and $\mathrm{M}_{0}$ is the initial weight of the dry HFFs.

\subsubsection{In vitro permeation studies}

To assess the ability of MTX to diffuse through the hydrated HFFs, the permeability of selected HFFs (chosen based on the results from the previous tests) to MTX was evaluated using Franz-type diffusion cells as previously described [37]. Briefly, HFFs were hydrated in $20 \mathrm{ml}$ of $10 \mathrm{mM}$ Phosphate buffer saline (PBS, $\mathrm{pH}=7.4$ ) for $24 \mathrm{~h}$ at room temperature. The hydrated HFFs were cut into circular portions and then mounted onto the modified Franz-type diffusion cells (Permegear Inc., Hellertown, PA, USA), with an orifice diameter of $15 \mathrm{~mm}$ 
(effective diffusional area $1.77 \mathrm{~cm}^{2}$ ) and receptor volume of approximately $12 \mathrm{ml}$. The diffusion cells were placed on a diffusion drive console to provide synchronous stirring at 600 $\mathrm{RPM}$ and thermostated at $37 \pm 1^{\circ} \mathrm{C}$. After equilibration for $2 \mathrm{~h}$, aliquots of $0.5 \mathrm{ml}$ of the MTX aqueous solution (14.8 $\mathrm{mg} / \mathrm{ml}$, calculated as MTX base) were applied into the donor compartment of each diffusion cell which corresponds to a dose of $4.2 \mathrm{mg} / \mathrm{cm}^{2}$. The donor compartments were covered with laboratory Parafilm $\mathrm{M}^{\circledR}$ to avoid evaporation of PBS or the applied MTX solution throughout experimentation. At pre-determined time intervals up to 24 h, a $300 \mu \mathrm{L}$ sample was collected via the side-arm of the Franz cell and then immediately replenished with an equivalent volume of release medium. MTX was quantified using the validated HPLC-UV method described in the analytical section. MTX permeation profiles were constructed and used to calculate MTX steady-state flux (reported as $\mu \mathrm{g} . \mathrm{h} / \mathrm{cm}^{2}$ ), and MTX permeated amounts $\left(\mu \mathrm{g} / \mathrm{cm}^{2}\right)$, after $6 \mathrm{~h}$ and $24 \mathrm{~h}$, respectively.

\subsubsection{Fourier transform infrared spectroscopy}

Attenuated total reflectance (ATR) Fourier transform infrared (FTIR) spectroscopy was used to investigate the esterification reaction between PVA and CA and the intermolecular interactions among the HFFs components. The IR spectra of the original materials, the nonecrosslinked films and the HFFs were recorded using a Spectrum Two ${ }^{\mathrm{TM}}$ instrument (Perkin Elmer, Waltham, MA, USA). The IR spectra were recorded between 400 and $4000 \mathrm{~cm}^{-1}$ with a resolution of $4 \mathrm{~cm}^{-1}$. The obtained spectra were the result of averaging 64 scans.

\subsubsection{Scanning electron microscopy}

The morphology of the HFFs was evaluated by using scanning electron microscopy (SEM). Samples from selected HFFs were first swollen to equilibrium in DI water at room temperature, quickly frozen in liquid nitrogen and then further freeze-dried in a VirTis freeze drier (Advantage XL-70, SP industries, NY, USA) under vacuum at $42^{\circ} \mathrm{C}$ for at least $48 \mathrm{~h}$ until all the solvent was sublimed. Freeze-dried HFFs samples were then fractured carefully, and their surface morphology was studied using Quanta FEG 250 benchtop scanning electron microscope (SEM) (FEI, Hillsboro, OR, USA) at an acceleration voltage of 10-20 kV under high chamber pressure $\left(8 \times 10^{-5} \mathrm{mbar}\right)$ with standard SEM carbon tape as background. Before SEM observation, specimens of the HFFs were fixed onto aluminium stubs and coated at 2.5 
kV, 18 mA with gold for 45 s (POLARON E5150, Gold Sputter Coater, Quorum Technologies, East Sussex, UK).

\subsection{Preparation of the hydrogel-forming microneedle arrays (HFMN)}

HFMN arrays were prepared from the finalised aqueous polymeric blends (chosen based on the results from studies performed on the HFFs) using the micromoulding technique previously described [25,26,28]. Laser-engineered poly(dimethylsiloxane) (PDMS) micromoulds $\left(0.5 \mathrm{~cm}^{2}\right.$ surface area, $11 \times 11$ array, $800 \mu \mathrm{m}$ height, $300 \mu \mathrm{m}$ width, and $300 \mu \mathrm{m}$ interspacing at MN base) were employed to manufacture the HFMN arrays. Briefly, $0.4 \mathrm{~g}$ of the selected aqueous polymeric blends were carefully poured into the PDMS micromoulds, centrifuged for $15 \mathrm{~min}$ at $3500 \mathrm{RPM}$ and then dried at room temperature for $48 \mathrm{~h}$ (Figure 2A). After drying, HFMN arrays were cured in a pre-heated oven at $130 \pm 1^{\circ} \mathrm{C}$ for a pre-determined time (optimal crosslinking time) to induce an ester-based crosslinking between PVA and CA. Upon cooling, the HFMN arrays were gently removed from the PDMS micromoulds. The sidewalls formed by the moulding process were then removed using a pre-heated scalpel. The resultant HFMN were then stored in sealed aluminium bags with added silica gel pouches until further use.

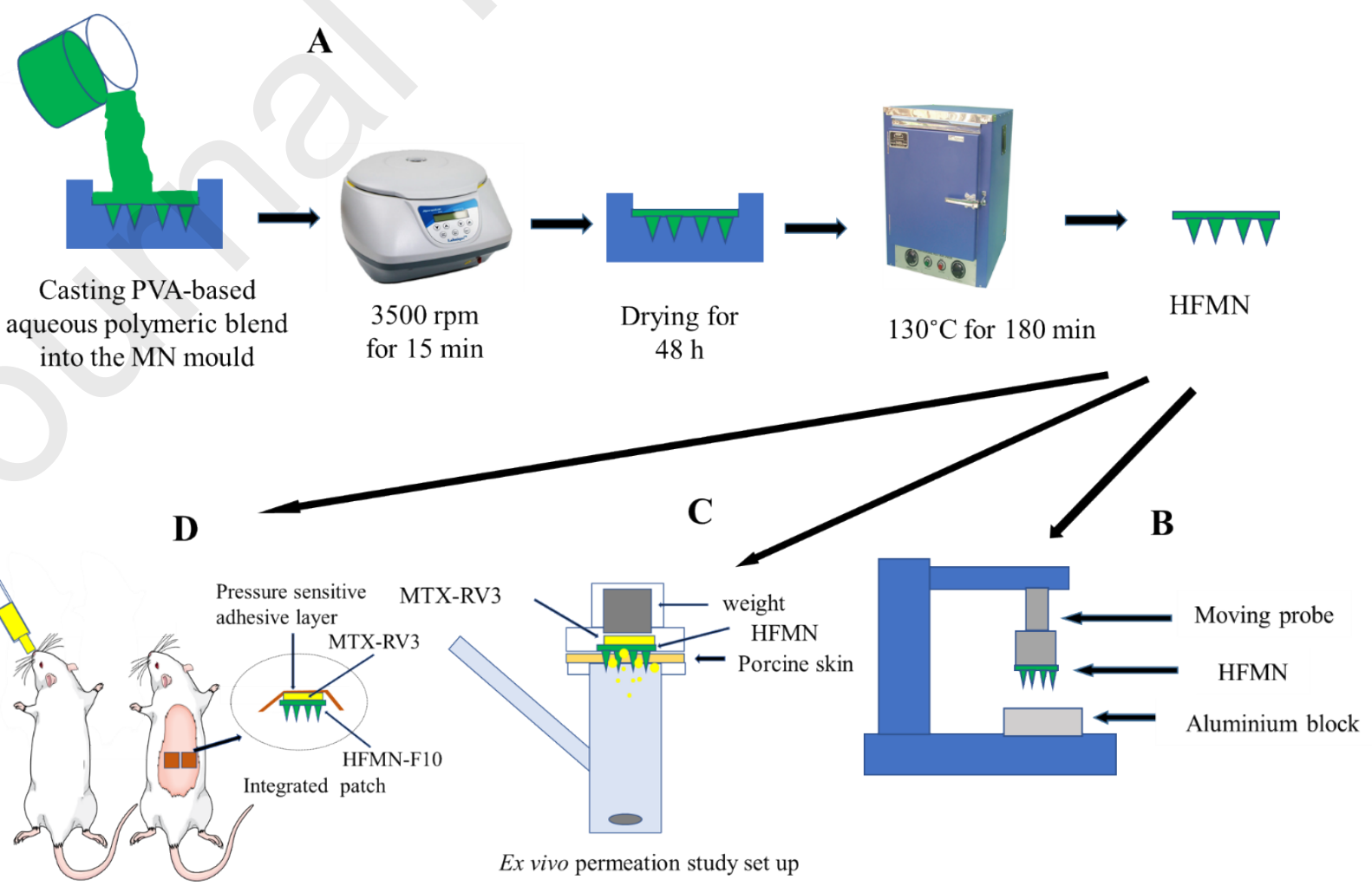


Figure 2. Schematic representation of preparation procedures for HFMN (A); experimental setup for measuring mechanical strength of HFMN (B); schematic diagram for the experimental setup for the ex vivo permeation studies (C); The experimental setup for the in vivo study, where two integrated patches were applied to the back of the rat and MTX aqueous solution administered orally (D).

\subsection{Assessment of the mechanical and insertion properties of the HFMN}

HFMN were subjected to a standard mechanical strength test using an axial compression load (32 N/ array) using the Texture Analyser as previously reported [26,28]. Briefly, the HFMN were attached to a cylindrical probe (cross-sectional area $1.5 \mathrm{~cm}^{2}$ ) and the Texture Analyser arm moved vertically downwards at a speed of $0.5 \mathrm{~mm} / \mathrm{s}$ and the HFMN was compressed against a flat aluminium block. A force of $32 \mathrm{~N}$ was applied for $30 \mathrm{~s}$ before the probe was moved upwards again. The lengths of individual needles in the HFMN arrays were measured before and after compression using a Leica EZ4W stereo microscope with an integrated camera (Leica Microsystems, Milton Keynes, UK). The percentage reduction of MN heights was calculated and reported.

Moreover, to study the insertion capabilities of the HFMN, full-thickness excised neonatal porcine skin (obtained from stillborn piglets) was used as previously reported [27,28]. The HFMN were compressed against the skin manually by applying an average insertion force of $20 \mathrm{~N} /$ array, for $30 \mathrm{~s}$ to mimic the application process in the clinical situation. The inserted MN were immediately visualised using an EX1301 optical coherence tomography (OCT) microscope (Michelson Diagnostics Ltd., Kent, UK). The obtained 2D images were analysed using the imaging software Image $J^{\circledR}$ (National Institutes of Health, Bethesda, USA). The scale of the image files obtained was 1.0 pixel $=4.2 \mu \mathrm{m}$. Finally, pores in the skin were visualised by using light microscope after being stained with methylene blue staining solution $(1 \mathrm{mg} / \mathrm{ml})$ in PBS (pH 7.4).

\subsection{Preparation and characterisation of methotrexate-containing reservoir (MTX-RV)}

After a preliminary study, a range of aqueous polymeric blends containing either PVP, PVP $360 \mathrm{~K}$ or HMPC in addition to glycerol (used as plasticiser) were selected to prepare various MTX-containing reservoirs (MTX-RV). The composition of the MTX-loaded aqueous polymeric blends of the respected MTX-RV is outlined in Table 2.

Table 2. Composition of the aqueous polymeric blends used to prepare MTX-RV.

\begin{tabular}{cccc}
\hline & \multicolumn{3}{c}{ Formulae (100 g) } \\
\cline { 2 - 4 } Formulae code & MTX-RV1 & MTX-RV2 & MTX-RV3 \\
\hline Ingredients & \multicolumn{3}{c}{$\%$ w/w } \\
\hline
\end{tabular}




\begin{tabular}{cccc}
\hline MTX & 3.3 & 3.3 & 3.3 \\
Glycerol & 10 & 5 & 5 \\
PVP & 30 & - & - \\
PVP K90 & - & 15 & - \\
HPMC & - & - & 7.5 \\
DI water & 56.7 & 76.7 & 83.2 \\
\hline
\end{tabular}

To prepare drug-free PVP and PVP 360K-based aqueous blends, the required amounts of the selected polymers was suspended in DI water and left for hydration overnight at room temperature while being shaken at 40 RPM until clear homogenous aqueous blends were formed. To prepare a drug-free HMPC-based aqueous blend, 7.5 g of HPMC was suspended in $40 \mathrm{~g}$ of pre-heated DI water to $90 \pm 1^{\circ} \mathrm{C}$, which was then vortexed for $10 \mathrm{~min}$ and subsequently another $40 \mathrm{~g}$ of cold DI water was added. The resultant suspension was left for hydration at room temperature, whilst being shaken at 40 RPM overnight until a clear homogenous aqueous blend was produced. The required amount of glycerol was then added to each aqueous polymeric blend. MTX was then added at 3.3\% w/w (equivalent to $3 \% \mathrm{w} / \mathrm{w}$ MTX base), and the final weight of each aqueous blend was adjusted to $100 \mathrm{~g}$ by DI water. The components were hand-mixed and homogenised using a stainless-steel spatula and then centrifuged at 3500 RPM for 15 min to remove any air bubbles. An aliquot (10 g) from each aqueous polymeric blend was slowly cast into the in-house made mould that was previously used in section 2.2. Since MTX is a photosensitive drug, the cast blends were dried away from light in a closed pre-heated oven at $37 \pm 1^{\circ} \mathrm{C}$ for $48 \mathrm{~h}$ to form large MTX-RV, which were then cut into smaller segments $(10 \times 10 \mathrm{~mm})$ and stored in a sealed aluminium bag until further use. MTX-RV were characterised for their density (which was calculated as the drug reservoir mass (g) per drug reservoir volume $\left(\mathrm{cm}^{3}\right)$ ), drug content (using the validated HPLC-UV method described in the analytical section), moisture content (using TGA, Q500, TA Instruments ${ }^{\mathrm{TM}}$ New Castle, Delaware, USA) and dissolution time. Dissolution time was determined by suspending 10 x $10 \mathrm{~mm}$ segments of each MTX-RV in $20 \mathrm{ml}$ of $10 \mathrm{mM}$ PBS solution (pH 7.4), maintained at $37 \pm 1^{\circ} \mathrm{C}$ and stirred with a magnetic bar at $200 \mathrm{RPM}$. The time for complete dissolution was recorded.

\subsection{Ex vivo permeation studies}


The diffusion of MTX from a selected MTX-RV (the finalised formula) through a selected HFMN (the finalised formula) and across a dermatomed neonatal porcine skin was investigated ex vivo using the modified Franz diffusion cells, as previously described [26,28]. Briefly, the neonatal porcine skin was excised and trimmed to a thickness of $350 \mu \mathrm{m}$ using an electric dermatome. To preserve the skin's mechanical and barrier properties without inducing biological decomposition and structural changes, the skin samples were stored in aluminium foil at $-20^{\circ} \mathrm{C}$ until further use [39-41].

Before use and to avoid hair interference with HFMN insertion, the skin was bathed in PBS (pH 7.4) to thaw and then, once defrosted, the hair was carefully shaved. Sections of skin were cut by a scalpel to match the diameter of the Franz cell donor compartments and carefully affixed to the donor compartment on the stratum corneum side using cyanoacrylate adhesive (Loctite, Dublin, Ireland), rendering the stratum corneum available for HFMN application. This was then placed on top of dental wax, to give the skin support, and the HFMN were then inserted into the centre of the skin section, using a plunger of $5 \mathrm{ml}$ disposable syringe at a force of approximately $20 \mathrm{~N} /$ array. The finalised MTX-RV (which was trimmed to $0.5 \mathrm{~cm}^{2}$ size using a paper puncher) was then placed on the top of the HFMN with $20 \mu \mathrm{L}$ of water to initiate adhesion. A tubular stainless-steel weight was then placed on the top of the integrated patch (Figure 2C). At predetermined time intervals, $300 \mu \mathrm{L}$ samples were collected via the side-arm of the Franz cell and the receiver compartment immediately replenished with an equivalent volume of release medium. As a control, MTX permeation from its finalised MTX-RV through the skin without using HFMN (by passive diffusion) was also evaluated in the same conditions. MTX was quantified using the validated HPLC-UV method described in the analytical section. MTX permeation profiles were constructed and used to calculate MTX steady-state flux and the permeated amounts at $24 \mathrm{~h}$.

\subsection{In vivo studies}

This study was conducted according to the policy of the Federation of European Laboratory Animal Science Associations and the European Convention for the protection of vertebrate animals used for experimental and other scientific purposes, with the implementation of the principles of the 3Rs (Replacement, Reduction and Refinement). The study was performed under Project Licence no. 2794 and Personal Licence no. 1747 at the 
Biological Services Unit, Queens University Belfast, after obtaining ethical permission from the Queen's University Belfast Animal Welfare and Ethics Review Board.

Based on the outcomes of the in vitro and ex vivo studies, both finalised MTX-RV and HFMN were combined to form an "integrated patch" to be tested in vivo using healthy female Sprague Dawley rats. The rats $(n=14)$, aged $12-14$ weeks and weighed $221.25 \pm 26.94 \mathrm{~g}$ were purchased from (Envigo Holdings, Inc., UK) and were allowed to acclimatise to laboratory conditions for at least 7 days before the beginning of the experiments. The rats were split into two cohorts. In the first cohort $(\mathrm{n}=6)$, which used as a control, each rat received $0.5 \mathrm{ml}$ an oral aqueous solution of MTX $(1.2 \mathrm{mg} / \mathrm{ml}$, calculated as MTX base $)$. This corresponds to a dose of $2.66 \pm 0.25 \mathrm{mg} / \mathrm{kg}$. This dose was selected because it gives a comparable area-under-the-curve (AUC) to the AUC in humans after giving the same dose $(2.5 \mathrm{mg})$ orally, thus a comparable systemic exposure would be expected [42].

The second cohort $(n=8)$ was used to apply the integrated patch. To minimize the interference between the rats' hair and HFMN, electric hair clippers were used to remove the bulk hair, and then a depilatory cream was applied to remove any residual hair. Skin barrier was then allowed to recover for $24 \mathrm{~h}$ before patch application. To facilitate the patch application, the rats were sedated using gas anaesthesia $(2-4 \% \mathrm{v} / \mathrm{v}$, isoflurane) in oxygen. Subsequently, each rat received two of the integrated patches. Each patch contains approximately $(0.6 \pm 0.13 \mathrm{mg})$ MTX (calculated as MTX base). This equates to MTX dose of $5.23 \pm 0.54 \mathrm{mg} / \mathrm{kg}$. The patches were applied manually to the rats` back (Figure 2D) by first inserting the HFMN into the skin and then applying an aliquot of DI water $(20 \mu \mathrm{L})$ to its centre to facilitate the MTX-RV adhesion, which was immediately applied on top of the HFMN to form an integrated patch. To secure the integrated patch in place, a pressure-sensitive adhesive tape (Microfoam ${ }^{\mathrm{TM}}$ Surgical Tape, 3M, Bracknell, UK) was applied as well as an adhesive thin film (Tegaderm ${ }^{\mathrm{TM}}$, 3M, St Paul, Minnesota, USA). Additionally, to further secure HFMN, Kinesiology ${ }^{\mathrm{TM}}$ tape (ProWorks Corporation, Corvallis, USA) was then gently wrapped around the back and abdomen of each rat. The integrated patches were removed after $24 \mathrm{~h}$. It is important to note that rats often try to remove the applied patches, thus some of the patches may be displaced or removed leading to a smaller number of replicates in the second cohort. To avoid such scenarios, two patches were applied on the back of each rat and two extra rats were used, thus, the total number of rats in this cohort become $(n=8)$. Additionally, in the second cohort, the total loaded MN dose was twice that of the oral dose because the HFMN may not deliver more than $50 \%$ of the loaded dose $[26,28]$. 
Blood samples $(\sim 200 \mu \mathrm{L})$ were collected via tail vein bleeds at pre-defined time intervals of 1, 2, 3, 6, 24, 27 and $48 \mathrm{~h}$. Under the Project Licence, a staggering study design was employed: rats were bled at a maximum of twice daily and at least 3 blood samples were collected for each time point. These samples were processed using a dried blood spot (DBS) technique, as previously described [43] and analysed using the validated HPLC-MS-MS method described in the analytical section.

To evaluate the safety of our novel drug delivery system, the HFMN arrays from the integrated patches were carefully inspected before and after application to the skin to assess their integrity, as well as monitoring rats' skin at the application site over $24 \mathrm{~h}$ following patch removal for any reactions.

\subsection{Pharmaceutical analysis}

\subsubsection{HPLC-UV method}

For quantification of MTX from the in vitro samples, a reversed-phase highperformance liquid chromatography (HPLC-UV) method was used. This method was adopted from Begas et al. [44], with minor modification and then validated as per the International Conference on Harmonization (ICH) guidelines. The analysis was performed on an Agilent 1220 Infinity LC system (Agilent Technologies UK Ltd., Stockport, UK) with a Kinetex ${ }^{\circledR} 5$

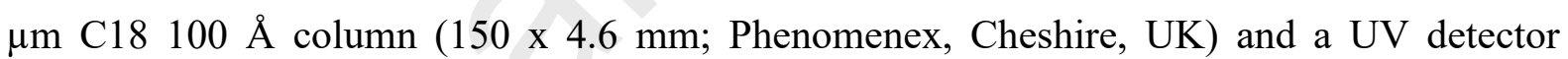
operated at $303 \mathrm{~nm}$. The column temperature was kept at $30 \pm 1{ }^{\circ} \mathrm{C}$. The separation was performed using a mobile phase which consisted of $20 \mathrm{mM}$ sodium acetate buffer ( $\mathrm{pH}$ 3.6): acetonitrile (90: $10 \mathrm{v} / \mathrm{v})$, that was pre-filtered through a $0.2 \mu \mathrm{m}$ pore size filter (Alltech, Deerfield, IL), and then degassed by an online degassing system and delivered at a flow rate of $1 \mathrm{ml} / \mathrm{min}$. The run time was $7 \mathrm{~min}$. The injection volume was $20 \mu \mathrm{L}$. Specificity was shown by comparing the chromatograms of blank and spiked samples. Inter- and intraday accuracy and precision were investigated and limit of detection (LOD) and limit of quantification (LOQ) were calculated based on the residual standard deviation of the regression line of the mean calibration curve plot.

\subsubsection{HPLC-MS-MS method}


MTX and its polyglutamates $\left(\mathrm{MTX}^{-} \mathrm{PG}_{1-5}\right)$ were quantified in blood samples collected from the in vivo study by employing an HPLC-MS-MS method adapted from Hawwa et al. [43]. The HPLC system used consisted of a Waters Alliance 2795 Separation Module coupled with a Waters Quattro Premier XE tandem quadrupole mass spectrometer (MS-MS) (Micromass, Manchester, UK), equipped with an electrospray ionisation (ESI) source. The instrument was operated in positive ion mode. An Atlantis T3-C18 column [150 x $2.1 \mathrm{~mm}$ (I.D.); particle size, $3 \mu \mathrm{m}$; Waters (Waters Corp. UK, Herts, UK)] protected with a guard cartridge [20 mm x $2.1 \mathrm{~mm}$; particle size, $3 \mu \mathrm{m}$; Waters] was used as a stationary phase. The HPLC system and the MS-MS were both controlled by MassLynx 4.0 Software with the QuanLynx Application Manager. The mobile phase consisted of $10 \mathrm{mM} \mathrm{NH} \mathrm{HCO}_{3}$ buffer adjusted to $\mathrm{pH} 7.5$ using formic acid (A) and acetonitrile (B). separation of the individual MTX$\mathrm{PG}_{1-5}$ was achieved using linear gradient elution from $0 \% \mathrm{v} / \mathrm{v}$ to $20 \% \mathrm{v} / \mathrm{v}$ mobile phase $\mathrm{B}$ over $20 \mathrm{~min}$ (at a flow rate of $0.15 \mathrm{ml} / \mathrm{min}$ ). After $20 \mathrm{~min}$, the mobile phase was returned to $100 \%$ mobile phase A and re-equilibrated for $10 \mathrm{~min}$. The column temperature was maintained at 30 $\pm 1^{\circ} \mathrm{C}$ and the autosampler at $4^{\circ} \mathrm{C}$. The method was validated for specificity, inter- and intraday accuracy and precision as per ICH guidelines. The limit of detection (LOD) and limit of quantification (LOQ) were again calculated based on the residual standard deviation of the regression line of the mean calibration curve plot.

\subsection{Calculation of MTX pharmacokinetic parameters in rats}

Data collected from analysing blood samples from the in vivo study was used to construct $\mathrm{MTX}-\mathrm{PG}_{1-5}$ pharmacokinetic profiles using the population pharmacokinetic approach. Non-compartmental pharmacokinetic analysis was carried out using Microsoft ${ }^{\circledR}$ Excel $^{\circledR} 2016$ (Microsoft Corporation, Redmond, USA) with PKsolver add-in [45]. The curve of drug concentration $v s$ time profiles were created in each case. The maximum drug concentration $\left(\mathrm{C}_{\max }\right)$, the time of maximum concentration $\left(\mathrm{T}_{\max }\right)$, the AUC from time zero $(\mathrm{t}=$ $0)$ to the last experimental time point $(\mathrm{t}=48 \mathrm{~h})\left(\mathrm{AUC}_{0-48}\right)$ and the AUC from time zero $(\mathrm{t}=0)$ to infinity ( $\mathrm{AUC}_{0 \text {-Inf }}$ ) were all calculated. The relative bioavailability (FR) of MTX from the integrated patch was calculated using Equation 3.

$$
\mathrm{FR}=\frac{\mathrm{AUC}(\mathrm{IP}) \times \mathrm{Dose}(\mathrm{PO})}{\mathrm{AUC}(\mathrm{PO}) \times \mathrm{Dose}(\mathrm{IP})} \quad \text { Equation } 3
$$

Where AUC (IP) is the area -under the curve (AUC 0 -Inf) obtained following MTX application using the integrated patch; $\mathrm{AUC}(\mathrm{PO})$ is the area -under the curve (AUC ${ }_{0 \text {-Inf }}$ ) 
obtained following MTX administration by the oral route; Dose (PO) is the MTX dose administered bythe oral route, and Dose (IP) is MTX dose applied using is the integrated patch.

\subsection{Statistical analysis}

All data were expressed as means \pm standard deviation (SD), $(\mathrm{n} \geq 3)$. Statistical analysis was performed using GraphPad Prism ${ }^{\circledR}$ version 6 (GraphPad Software, San Diego, California, USA). Where appropriate, an unpaired t-test was carried out for comparison of two groups. Kruskal Wallis test with post-hoc Dun`s test was performed for comparison of multiple groups. In all cases, $p<0.05$ was the minimum value considered acceptable for rejection of the null hypothesis.

\section{Results and Discussion}

\subsection{Preparation and characterisation of the HFFs}

HFFs were prepared from various aqueous polymeric blends consisting of carefully selected polymers and a crosslinking agent. The criteria were set to use polymers and crosslinking agent that are (i) biocompatible, (ii) have functional groups that can be crosslinked using a simple and cost-effective method without using toxic chemical mediators or organic solvents, (iii) the degree of crosslinking can be controlled, and (iv) importantly, upon crosslinking, they form chemically MTX-compatible crosslinked materials that allow MTX diffusion without causing precipitation.

PVA is a water-soluble polymer with an excellent profile in terms of its low toxicity and biocompatibility [46-48]. This polymer is widely used in fabricating advanced biomedical systems and devices, including wound dressing [49,50], contact lenses [51] and MNs [52-56]. Importantly, PVA is an inert polymer with repeating hydroxyl groups, which makes it an ideal candidate for physical and chemical crosslinking [55,56] [32]. Therefore, it was selected to form the polymeric base.

Previously, a physical crosslinking, by multiple freeze-thaw cycles, has been used to prepare HFMN from PVA [54,55,57]. Unfortunately, this method is tedious and timeconsuming. For example, preparing a PVA-based HFMN requires three repetitions of $24 \mathrm{~h}$ of freeze-thaw cycles [55]. Additionally, the produced HFMN by these methods have a weak 
structure and the degree of crosslinking is difficult to control $[54,55]$. In contrast, chemical crosslinking is simple, fast and cost-effective and the degree of crosslinking can be controlled readily [32]. Nevertheless, it has always been avoided to prepare PVA-based HFMN due to concerns over the remaining traces of toxic crosslinker [55,57]. CA is a non-toxic, weak, organic tricarboxylic acid that is widely used as a food additive (E330) and as an excipient in the pharmaceutical formulation [58]. It can react with the hydroxyl groups in the PVA by heating at $130^{\circ} \mathrm{C}$ for 40 min without using any toxic chemical mediators or organic solvents via a so-called "green route" to form insoluble polymeric materials. If it is used at an appropriate ratio with PVA, it does not produce surplus carboxylic groups, thus, the crosslinked materials will have neutral or near neutral $\mathrm{pH}$ [32]. Therefore, in this study, the HFMN were prepared by chemical crosslinking method using CA as crosslinking.

HFFs were prepared from various polymeric blends containing CA and PVA alone or with other polymers/solvents to select the best blend composition to fabricate useful HFMN to deliver MTX transdermally in a sustained manner. The HFFs were assessed for (i) their mechanical properties by measuring $\mathrm{F}_{\max }$ and thickness, (ii) swelling properties by measuring the percentage of swelling $(\% \mathrm{~S})$ over time and $\% \mathrm{SE}$, and (iii) the percentage of insoluble polymers recovered after swelling by measuring \%GF. The favoured polymeric blends were set to be, those which can demonstrate the following characteristics: good mechanical properties, thus they are neither flexible nor brittle and $\mathrm{F}_{\max }>23.55 \mathrm{~N} / \mathrm{cm}^{2}$ [26], reasonable swelling in water $(\% \mathrm{SE}>200)$, and maximal insoluble polymers recovery from the skin, i.e. high \%GF. The effect of crosslinking time of the HFFs properties was also investigated. Results are reported in Table 3 and 4, respectively and Figure 3. Following on from these studies, HFFs prepared from the finalised blend composition and crosslinking time were tested for their permeability to MTX (in vitro) to evaluate the chemical compatibility with MTX and their ability to allow the drug to diffuse through without precipitation or complex formation.

\subsubsection{Mechanical properties of the HFFs}

The mechanical properties of the hydrogel-forming materials in the dry state is a key factor in preparing useful HFMN capable of piercing the outermost layer of the skin [25]. Therefore, it was first evaluated by measuring the maximum break/bend force $\left(\mathrm{F}_{\max }\right)$, which give an insight for the mechanical properties (strength) of the HFFs, thus the HFMN, the higher 
$F_{\max }$, the stronger the HFFs [34]. HFFs thickness can also influence the HFFs mechanical properties. Therefore, it was measured.

The study was initiated by evaluating the effect of the composition of the polymeric blend on the mechanical properties of the HFFs. As can be seen from Table 3, while HFFs prepared from F1 and F2 (containing CA and PVA at 5 and $10 \% \mathrm{w} / \mathrm{w}$, respectively) were thin and too flexible to be tested. HFFs prepared from F5 (containing CA and PVA 15\% w/w with added glycerol at $5 \% \mathrm{w} / \mathrm{w}$ ) were flexible and soluble. HFFs could not be prepared from F6 or F7 (containing CA and PVA 15\%, w/w with added PEG 400 and PEG 10,000, respectively) due to incompatibility, where the polymeric blend was inhomogeneous and separated into two layers on rest. Therefore, polymeric blends F1, F2, F5, F6, and F7 were discarded.

HFFs were then prepared from the other polymeric blends, namely F3, F4, F8, F9 and F10 showed promising mechanical properties. The thickness of these HFFs ranged between 0.67 and $0.99 \mathrm{~mm}$. The HFFs thickness was dependent upon the polymer content in the polymeric blend. $F_{\max }$ of the HFFs varied from 6.1 to $29.0 \mathrm{~N} / \mathrm{cm}^{2}$. HFFs prepared from $\mathrm{F} 3$ (contains CA and PVA added at 1.5 and $15 \% \mathrm{w} / \mathrm{w}$, respectively) and F4 (contains CA and PVA added at 3 and $15 \% \mathrm{w} / \mathrm{w}$, respectively) showed a degree of hardness ( $\mathrm{F}_{\max }$ was 6.1 and 7.2 $\mathrm{N} / \mathrm{cm}^{2}$, respectively), but those were not hard enough to produce useful HFMN. Additionally, it was observed, that upon crosslinking, the HFFs prepared from those polymeric blends (F3 and F4) folded and deformed. To keep them flat, we had to entrap the HFFs in-between two layers of metal mesh.

Table 3. The characteristics of HFFs prepared from various aqueous PVA-based polymeric blends. Data is reported as means $\pm \mathrm{SD}, \mathrm{n} \geq 3$.

\begin{tabular}{ccccc}
\hline Formulae code & Thickness $(\boldsymbol{\mu m})$ & $\mathbf{F}_{\mathbf{m a x}}\left(\mathbf{N} / \mathbf{c m}^{\mathbf{2}}\right)$ & $\mathbf{\%} \mathbf{S E}$ & $\mathbf{\% G F}$ \\
\hline F1 & $0.25 \pm 0.11$ & Flexible & $\mathrm{ND}$ & $\mathrm{ND}$ \\
F2 & $0.42 \pm 0.22$ & Flexible & $\mathrm{ND}$ & $\mathrm{ND}$ \\
F3 & $0.67 \pm 0.08$ & $6.1 \pm 2.9$ & $1093.0 \pm 95.2$ & $71.1 \pm 0.7$ \\
F4 & $0.74 \pm 0.06$ & $7.2 \pm 2.2$ & $721.3 \pm 40.7$ & $77.3 \pm 1.4$ \\
F5 & $\mathrm{ND}$ & Flexible & Dissolved & ND \\
F6 & $\mathrm{ND}$ & $\mathrm{ND}$ & $\mathrm{ND}$ & $\mathrm{ND}$ \\
F7 & $\mathrm{ND}$ & $\mathrm{ND}$ & $\mathrm{ND}$ & $\mathrm{ND}$ \\
F8 & $0.87 \pm 0.11$ & $16.5 \pm 2.7$ & $625.6 \pm 66.6$ & $73.8 \pm 0.3$ \\
F9 & $0.92 \pm 0.10$ & $18.5 \pm 2.5$ & $593 \pm 24.7$ & $74.9 \pm 1.2$ \\
F10 & $0.99 \pm 0.04$ & $29.0 \pm 3.6$ & $492.1 \pm 33.4$ & $79.7 \pm 1.0$ \\
\hline
\end{tabular}

ND means not determined.

Interestingly, HFFs prepared from polymeric blends F8, F9 and F10 were flat and exhibited significantly higher $\mathrm{F}_{\max }$ in comparison with $\mathrm{F} 3$ and $\mathrm{F} 4(p<0.001) . \mathrm{F}_{\max }$ of the 
respected HFFs was dependent on PVP concentration in the polymeric blend. The higher the PVP concentration, the higher $F_{\max }$. For instance, $F_{\max }$ for HFFs prepared from F8 was 16.5 $\mathrm{N} / \mathrm{cm}^{2}$. This is around 2.5-fold higher than F3. However, HFFs prepared from F10 had $\mathrm{F}_{\max }$ equal to $29.0 \mathrm{~N} / \mathrm{cm}^{2}$, which is approximately 5 -fold higher than F3. This can be ascribed partly to the increased polymeric content in the polymeric blend [31] and to the plasticising effect of PVP and the potential of forming hydrogen bonds between $-\mathrm{OH}$ groups of PVA and $\mathrm{C}=\mathrm{O}$ groups of PVP, thereby increasing the cross-linking degree [36,59]. It is worth mentioning that none of the HFFs was broken during the mechanical strength testing but rather bent, indicating good mechanical properties and potential suitability for fabricating strong HFMN arrays. Leading on from the results of this study, only F3, F4, F8, F9 and F10 were selected to move forward and subjected to further testing.

\subsubsection{Swelling kinetics and the percentage of gel fraction of the HFFs}

Swelling properties and robustness of the crosslinked materials are two essential parameters that could affect the performance of the HFMN and, therefore, its usefulness as a drug delivery system. Ideally, the crosslinked materials should swell markedly (high \%SE), so that, upon insertion, HFMN can rapidly absorb skin interstitial fluid to form continuous and unblockable conduits between the dermal microcirculation and an attached patch-like drug reservoir, allowing drug diffusion through the swollen hydrogel-forming materials [25,34]. Also, the crosslinking reaction needs to be efficient so that \%GF is maximal, thus the amount of polymer that may leach into the skin is minimal [25,35]. In terms of swelling, the selected HFFs (F3, F4, F8, F9 and F10) swelled markedly and rapidly. Half of the total swelling took place within the first $2 \mathrm{~h}$ from immersing the HFFs in DI water, except F3 which took a long time as can be seen from Figure 3A. 

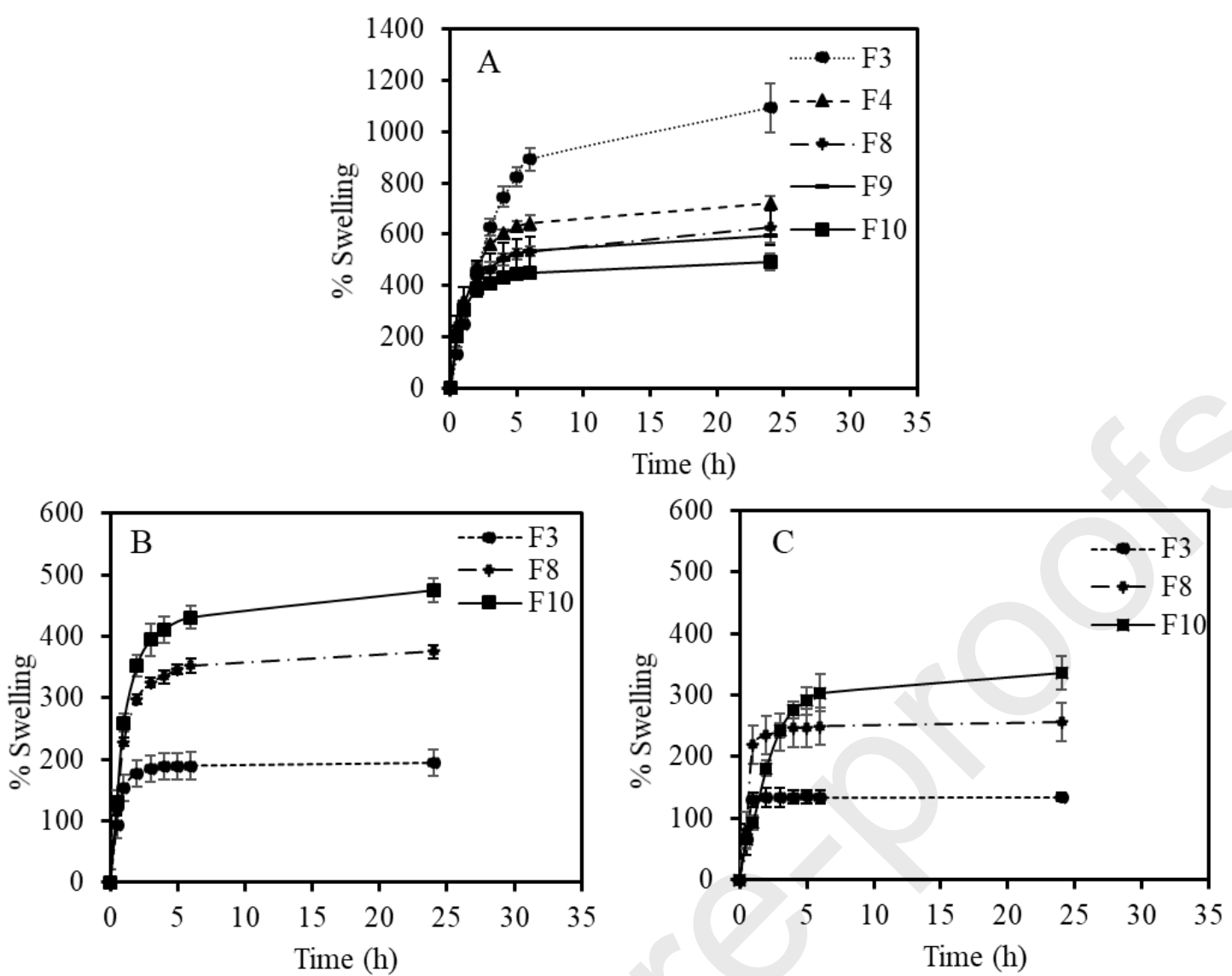

Figure 3. Swelling kinetics of selected HFFs crosslinked for $40 \min (\mathbf{A}), 120 \mathrm{~min}(\mathbf{B})$ or $180 \mathrm{~min}(\mathbf{C})$. Data is reported as means $\pm \mathrm{SD}, \mathrm{n}=3$.

The \%SE ranged between 1093.0\% (F3) and 492.1\% (F10) (Table 3). HFFs prepared from F8, F9 and F10 (containing PVP added at 5, 7.5, and 10\%, respectively) exhibited reduced $\% \mathrm{SE}$ in comparison with F3 and F4 (without PVP). The \%SE of the HFFs was again affected by PVP concentration in the polymeric blend. The higher the PVP concentration, the lower the $\%$ SE. For instance, \%SE for HFFs prepared from F8 (containing PVP added at 5\% w/w) was $625.6 \%$, while \%SE for HFFs prepared from F10 (containing PVP added at 10\% w/w) was 492.1\%. It was observed there was no significant difference $(p>0.95)$ in \%SE between F8 and F9.

In terms of $\% \mathrm{GF}$, it varied from $71.1 \%(\mathrm{~F} 3)$ to $79.7 \%$ (F10). Again, the polymeric blends F8, F9 and F10 (containing PVP) exhibited a significantly higher \%GF in comparison with F3 and F4 (without PVP) ( $p<0.001)$. F8 had a \%GF of $73.8 \%$, while F10 presented a $\% \mathrm{GF}$ of $79.7 \%$. Again statistically, there was no significant difference in \%GF between the HFFs prepared F8 and F9 $(p>0.95)$. The reduced $\% \mathrm{SE}$ and enhanced $\% \mathrm{GF}$ and $\mathrm{F}_{\max }$ are consistent with a physical crosslinking between PVA and PVP via forming hydrogen bonds between the $\mathrm{C}=\mathrm{O}$ groups from PVP and $-\mathrm{OH}$ groups from PVA $[59,60]$. 
Leading on the results of the accomplished tests, F8 and F10 appeared to be the most promising polymeric blends which could be used to fabricate useful HFMN, as they exhibited high $\mathrm{F}_{\max }$, moderate $\% \mathrm{SE}$ and relatively high \%GF in comparison with F3 and F4. Furthermore, F3, F4 and F9 were deemed unsuitable for fabricating HFMN and were discarded. While F3 and $\mathrm{F} 4$ showed relatively low $\mathrm{F}_{\max }$, low $\% \mathrm{GF}$ and deformed upon crosslinking, $\mathrm{F} 9$ did not exhibit statistically better performance in comparison with $\mathrm{F} 8$ in terms of $\mathrm{F}_{\max }, \% \mathrm{SE}$ and $\% \mathrm{GF}$.

Although the HFFs prepared from F8 and F10 showed higher \%GF in comparison with F3 and F4, they never reached $100 \%$, indicating that the crosslinking reaction was incomplete. Therefore, a further improvement was needed and so the effect of increasing the crosslinking time to 120 and 180 on the properties of HFFs prepared from F8 and F10 was investigated. F3 was used as a control to appreciate how PVP affects the properties of the HFFs when longer crosslinking time is implied. Results are outlined in Table 4. The swelling kinetics of the selected HFFs are presented in Figures 3(A and B).

Table 4. The characteristics of the HFFs prepared from F3, F8 and F10 blends and crosslinked in a preheated oven at $130 \pm 1{ }^{\circ} \mathrm{C}$ for 120 and $180 \mathrm{~min}$. Data is reported as means $\pm \mathrm{SD}, \mathrm{n} \geq 3$.

\begin{tabular}{ccccc}
\hline \multirow{2}{*}{ Crosslinking time (min) } & Formulae code & $\mathbf{F}_{\max }\left(\mathbf{N} / \mathbf{c m}^{2}\right)$ & \%SE & \%GF \\
\hline \multirow{2}{*}{120} & F3 & $12.1 \pm 2.5$ & $194.1 \pm 5.1$ & $94.8 \pm 1.0$ \\
& F8 & $29.8 \pm 2.7$ & $374.9 \pm 10.2$ & $89.3 \pm 1.6$ \\
& F10 & $37.4 \pm 2.3$ & $475.0 \pm 15.9$ & $82.1 \pm 1.9$ \\
\multirow{2}{*}{180} & F3 & $13.6 \pm 1.7$ & $134.5 \pm 6.4$ & $96.9 \pm 1.0$ \\
& F8 & $35.6 \pm 3.7$ & $255.9 \pm 6.4$ & $94.3 \pm 1.6$ \\
& F10 & $43.4 \pm 3.3$ & $340.6 \pm 14.9$ & $89.6 \pm 1.3$ \\
\hline
\end{tabular}

As can be seen from Table 4, increasing the crosslinking time from $40 \mathrm{~min}$ to 120 and then to $180 \mathrm{~min}$ resulted in a significant increase in \%GF of the selected HFFs $(p<0.001)$. $\%$ Gf trend displays a gradual increment in all HFFs. The longer the crosslinking time, the higher the \%GF. The highest \%GF were obtained when HFFs was crosslinked for 180 min. The \%GF of the HFFs prepared from F8 and F10 and crosslinked for 180 min were 94.3 and $89.6 \%$, respectively. These represent a significant improvement in \%GF in comparison with those HFFs crosslinked for only $40 \min (p<0.001)$. Furthermore, it was observed that the crosslinking time also affected the $\% \mathrm{SE}, \mathrm{F}_{\max }$ and swelling kinetics of the HFFs. Increasing the crosslinking time to 180 min led to a significant decrease in the \%SE of F8 and F10, which were 255.9 and $340.6 \%$, respectively $(p<0.05)$, indicating a moderate swelling capability. These represent approximately $23.9 \%$ and $31.7 \%$, respectively, of that reported for Gantrez ${ }^{\circledR}$ AN-139-based HFMN, which has \%SE equals to $1071 \pm 106 \%$ [26]. Thus, HFMN prepared 
those polymeric blends and crosslinked for 180 min should still imbibe skin interstitial fluid upon HFMN insertion.

In terms of the mechanical properties of the HFFs, $F_{\max }$ increased by increasing the crosslinking time. $F_{\max }$ for HFFs prepared from F8 and F10 and crosslinked for 180 min reached 35.6 and $43.4 \mathrm{~N} / \mathrm{cm}^{2}$, respectively. These were significantly $(p<0.001)$ higher than $F_{\max }$ (around $23.55 \mathrm{~N} / \mathrm{cm}^{2}$ ) for the HFFs prepared from Gantrez ${ }^{\circledR} \mathrm{AN}-139$ that is widely used to prepare HFMN [25]. Importantly, none of the HFFs prepared from F8 or F10 were broken while performing the break/bend strength testing but rather bending. This reflects not only the considerable mechanical strength of the HFFs material but also robustness and conformability. This is a paramount quality for the hydrogel-forming materials that are utilised in fabricating HFMN. If the materials too rigid, the HFMN MN may shatter and break while inserting into the skin, leaving insoluble polymers, which is highly undesirable. Also, the baseplate could break during insertion, due to the irregular topography of the skin surface on a microscopic scale and the macroscopic curvature of different body regions [25,34]. Leading on from the results from this study, $180 \mathrm{~min}$ was selected as the finalised crosslinking time, as it improved $\% \mathrm{GF}$ and $\mathrm{F}_{\max }$ significantly.

\subsubsection{HPLC-UV method validation}

In the in vitro and ex vivo experiments, MTX was quantified using a validated HPLCUV method as per ICH standard. MTX eluted at $5.1 \mathrm{~min}$ with no interference with any impurities from the analysed matrices. The detector response was linear in the range of 0.25 $100 \mu \mathrm{g} / \mathrm{ml}\left(\mathrm{R}^{2}>0.9999\right)$. The slope was 349.3 . The method showed excellent intra-day and inter-day accuracy ( $\mathrm{RE} \%<3.41 \%$ and $4.01 \%$, respectively) and precession (RSD $\%$ were $<2.86$ and $4.21 \%$, respectively) $(\mathrm{n}=6)$. The limit of detection $(\mathrm{LoD})$ was $0.083 \mu \mathrm{g} / \mathrm{ml}$ and the limit of quantitation (LoQ) was $0.25 \mu \mathrm{g} / \mathrm{ml}$.

\subsubsection{In vitro permeation studies}

This study was performed to evaluate the effect of the polymeric blend composition (F8 and F10) and the crosslinking time on the HFFs permeability to MTX (in vitro) using fully hydrated HFFs mounted onto Franz-diffusion cells. MTX permeation profiles through HFFsF8 and HFFs-F10 are presented in Figure 4. As can be seen from the graph, MTX exhibited 
almost a typical first-order release profile across both F8 and F10. Additionally, both HFFs exhibited excellent efficiency in delivering MTX. Over $66.6 \%$ of the applied dose $(4.2$ $\mathrm{mg} / \mathrm{cm}^{2}$ ) was delivered in all cases.
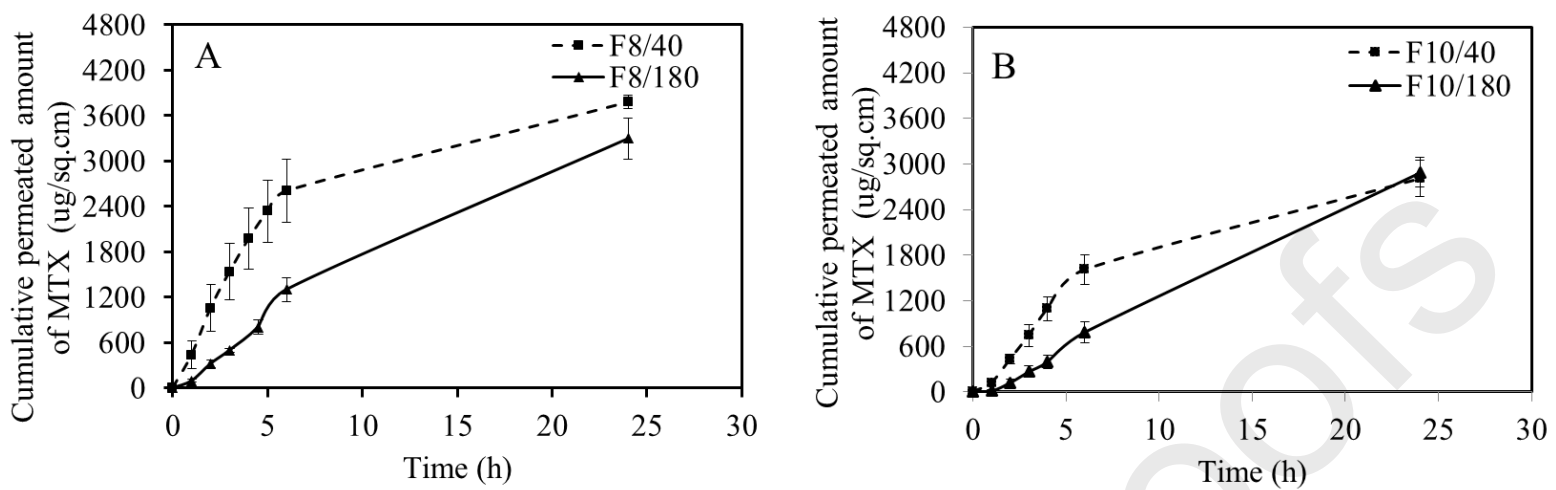

Figure 4. MTX permeation profiles through fully hydrated HFFs prepared from F8 crosslinked for either 40 min (F8/40) or $180 \mathrm{~min}(\mathrm{~F} 8 / 180)(\mathrm{A})$ and F10 crosslinked for either $40 \mathrm{~min}(\mathrm{~F} 10 / 40)$ or $180 \mathrm{~min}(\mathrm{~F} 10 / 180)$ (B). Data is reported as means $\pm \mathrm{SD}, \mathrm{n}=3$.

It was overserved that HFFs prepared from F10 (contains PVP added for the PVAbased aqueous blend at $10 \% \mathrm{w} / \mathrm{w}$ ) exhibited significantly lower steady-state flux in comparison with F8 (contained PVP added for the PVA-based aqueous blend at 5\%w/w). For example, the steady-state flux of MTX delivered through HFFs prepared from F10 and crosslinked for $40 \mathrm{~min}$ was $268.2 \mu \mathrm{g} . \mathrm{h} / \mathrm{cm}^{2}$. This is around $60 \%$ of the steady-state flux $\left(435.0 \mu \mathrm{g} . \mathrm{h} / \mathrm{cm}^{2}\right)$ of MTX delivered through HFFs prepared from F8.

The crosslinking time has a profound effect on the HFFs permeability to MTX. Increasing the crosslinking time to 180 min resulted in a significant decrease of MTX steadystate flux through both F8 and F10, which were almost half of the previously reported values. However, the amounts of MTX released over $24 \mathrm{~h}$ were not affected. For example, the amount of MTX released through HFFs prepared from F10 and F8 (crosslinked for 180 min) were 2889.5 and $3298.0 \mu \mathrm{g} / \mathrm{cm}^{2}$, respectively. These correspond to 68.5 and $78.2 \%$ of the applied dose $\left(4.2 \mathrm{mg} / \mathrm{cm}^{2}\right)$, respectively. This suggests that increasing the crosslinking time can affect the delivery rate but not the extent. It is worth mentioning that the undelivered MTX was adsorbed on the HFFs materials as they became yellowish once removed from Franz-diffusion cells at the end of the experiment.

Leading on the results of the accomplished tests on the HFFs so far, F8 and F10 crosslinked for 180 min can be used to fabricate useful HFMN arrays as they exhibited high $\mathrm{F}_{\max }$, moderate $\% \mathrm{SE}$, relatively high $\% \mathrm{GF}$ and allow MTX to permeate through efficiently in 
a sustained manner. Therefore, they were selected to move forward to use them to prepare HFMN arrays.

\subsubsection{FTIR spectroscopy}

Representative IR spectra for the individual materials; PVA, PVP and CA, noncrosslinked films and HFFs prepared from selected polymeric blends (F3 F8, F9 and F10) are illustrated in Figure 5. PVA spectrum (Figure 5A) shows broadband at $3291-3331 \mathrm{~cm}^{-1}$, indicating the presence of $\mathrm{OH}$ groups. $\mathrm{CA}$ spectrum shows a broad absorption band in the region between $2900-3400 \mathrm{~cm}^{-1}$, which can be attributed to the stretching vibration of the hydroxyl (-OH) from the carboxylic group. The band from 1689 to $1759 \mathrm{~cm}^{-1}$ is the typical absorption band for carbonyl $(\mathrm{C}=\mathrm{O})$ of the carboxylic group [32]. PVP spectrum shows a characteristic $\mathrm{C}=\mathrm{O}$ peak at around $1650 \mathrm{~cm}^{-1}$ and a broad peak at $3500 \mathrm{~cm}^{-1}$ that may be due to the presence of moisture in the material. The HFFs also showed all the characteristic peaks related to their components. It should be noted that F8 and F10 (contain PVP) showed additional peaks that is the characteristic to $\mathrm{C}=\mathrm{O}$ for $\mathrm{PVP}$, which were not observed in $\mathrm{F} 3$ (has no PVP). The intensity of this peak is proportional to the concentration of PVP in the polymeric blend.

It is worth mentioning that, when PVP and PVA were combined in F8 and F10, a shift in the PVP $\mathrm{C}=\mathrm{O}$ peak was observed even in the non-crosslinked films (Figure 5B). This shift is a characteristic of hydrogen bonds between the $\mathrm{C}=\mathrm{O}$ group from PVP and the $\mathrm{OH}$ group from PVA [59]. This is consistent with the plasticising effect of PVP in the films that have been described above. Moreover, this is in line with previous studies, which have indicated that physical crosslinking could happen between PVA and PVP upon heating their hydrogel mixture [61] [60]. When HFFs prepared from F10 were crosslinked at $130^{\circ} \mathrm{C}$ for different times, a crosslinking took place between the $\mathrm{COOH}$ from $\mathrm{CA}$ and the $-\mathrm{OH}$ groups from PVA (Figure 5D). The IR spectra of the HFFs showed apparent differences when compared with the non-crosslinked films F10 (Figure 5B).

Figure 5C shows the IR spectra of F10 that was crosslinked for various times (40, 120 and $180 \mathrm{~min}$ ). It was observed that the peak intensity at around $1720 \mathrm{~cm}^{-1}$ increased by increasing the crosslinking time. This peak can be attributed to the $\mathrm{C}=\mathrm{O}$ of the acid and the ester groups $[32,62]$. The intensity of the peak increases due to the formation of new ester bonds. This is consistent with the increased mechanical strength, higher \%GF and reduced 
$\% \mathrm{SE}$ of the corresponding HFFs that were described in the previous sections. The same characteristics were observed in other HFFs prepared from different polymeric blends (F3, F8, F9). However, only F10 results are shown in this work. It is worth mentioning that HFFs from all polymeric blends were transparent. Furthermore, it was noticed that HFF became slightly yellow when the crosslinking time was increased, which is possibly due to dehydration of CA and the formation of a double bond sensitive to oxidation, as previously suggested [32].

\subsubsection{Scanning electron microscopy (SEM)}

The effect of crosslinking time on the properties of the HFFs was evaluated by studying their morphology using SEM. Representative SEM images of fully hydrated and then lyophilised HFFs prepared from F10 are presented in Figure 5 (E and F). As can be seen, F10 crosslinked for $40 \mathrm{~min}$ showed a porous structure (Figure 5E). However, HFFs crosslinked for 180 min (Figure 5F) appeared as a solid surface, with a smaller pore size that could be barely noticed by SEM, even at high magnification $(20,000 \mathrm{x})$. We would expect to see this phenomenon in the hydrated HFMN prepared from the same polymeric materials and crosslinking times. This can be ascribed to a longer crosslinking time resulting in a significant increase in the crosslinking degree, therefore, more crosslink points were formed, and HFFs with much smaller pore size were produced [31]. These findings are in good agreement with the results of our studies reported in the previous sections (3.1.1- 3.1.4). It explains the high

values of $\mathrm{F}_{\max }, \% \mathrm{SE}, \% \mathrm{GF}$ and MTX-steady-state flux for HFFs prepared from F10 and crosslinked for $180 \mathrm{~min}$, in comparison with those reported for HFFs made from F10, but crosslinked for 40 min only ( $p<0.0001)$. Similar results were also observed among other HFFs prepared from F8. 
$\mathbf{A}$

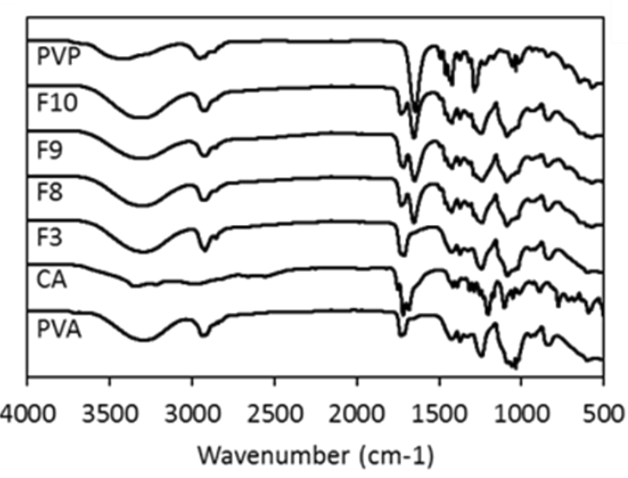

D

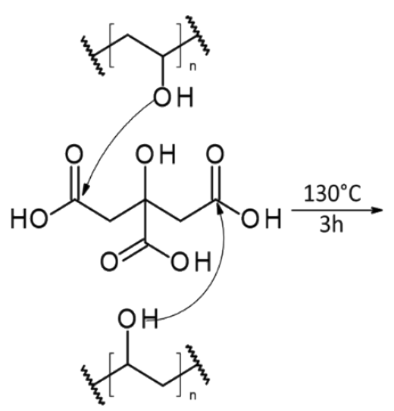

B

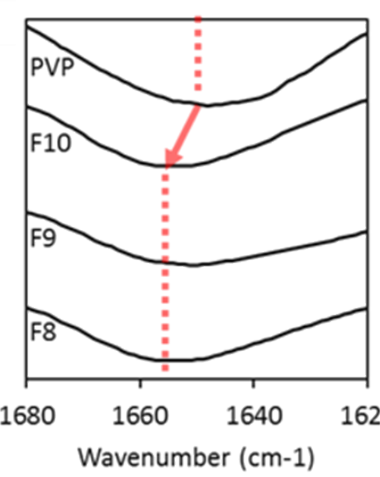

C

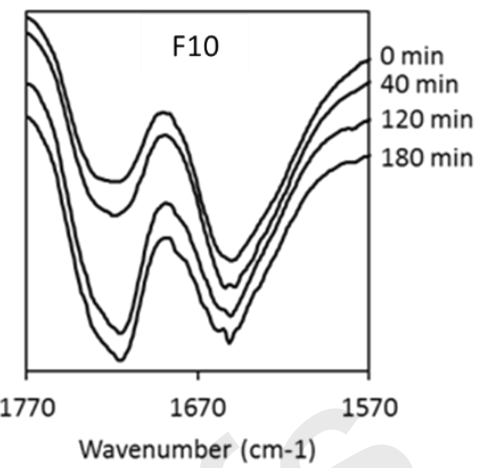

$\mathbf{F}$

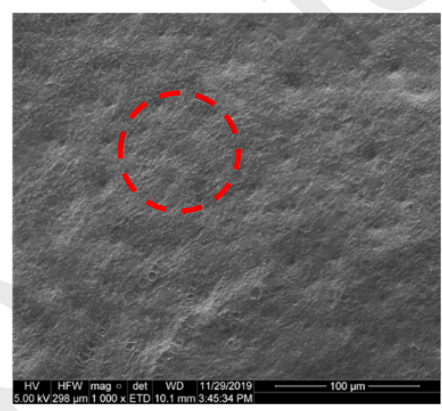

Figure 5. Representative FTIR spectra of PVP, PVA, CA and non-crosslinked films prepared from gel formulations F3, F8, F9 and F10 (A); the carbonyl region from FTIR spectra of PVP and non-crosslinked films prepared from gel formulations F8, F9 and F10 (B); the carbonyl region from FTIR spectra of HFFs made from gel formulation F10 that was crosslinked for 40,120 and $180 \mathrm{~min}$. (C); the likely structure of intermolecular crosslinked PVA with CA (D), representative samples of SEM images (magnification power of 20,000x) showing the morphological differences and the porous structure variation between HFFs-F10 crosslinked for $40 \mathrm{~min}$, where clear wide pores could be observed (E) and that crosslinked for $180 \mathrm{~min}$, where small pores could be observed (F).

\subsection{Preparation and characterisation of HFMN arrays}

HFMN were successfully prepared from the optimal polymeric blends F10 (HFMNF10) and F8 (HFMN-F8) and crosslinked for $180 \mathrm{~min}$ (the optimal crosslinking time). Representative samples of microscopic images for both HFMN are shown in Figure 6 (A and B). As can be seen, both HFMN looked identical in appearance and dimensions. However, HFMN-F10 has a darker yellowish colour, which is probably related to the higher PVP content in comparison with HFMN-F8. Each array had a baseplate with a total surface area of $0.5 \mathrm{~cm}^{2}$ and consisted of $11 \times 11$ needle arrays, each needle measuring $729.5 \pm 50.2 \mu \mathrm{m}$ in height and $300 \mu \mathrm{m}$ in width at the baseplate. The interspace between $\mathrm{MN}$ was around $300 \mu \mathrm{m}$. The dimensions of the produced HFMN were generally in good agreement with the theoretical aspects of the PDMS mould. After removing the sidewalls, the mean weight for HFMN-F8 and HFMN-F10, were of the magnitude of $18.2 \pm 1.5$ and $20.2 \pm 2.0 \mathrm{mg}$, respectively. 
A
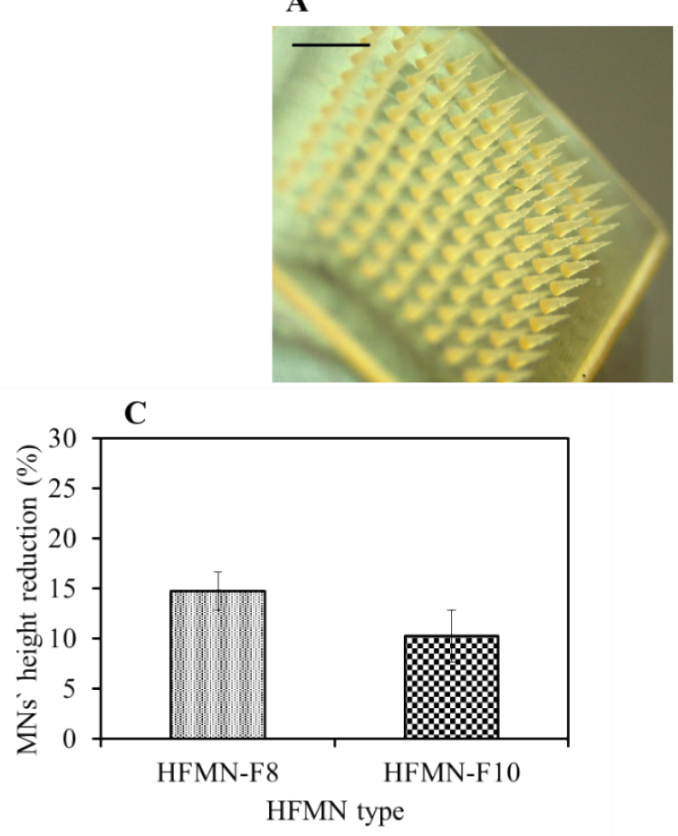

E

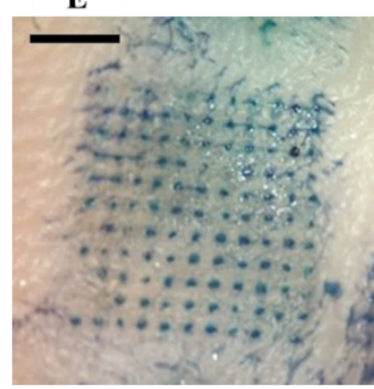

B

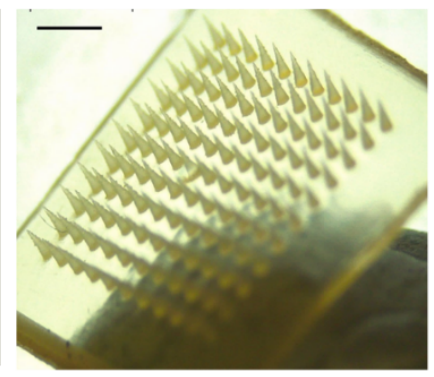

D

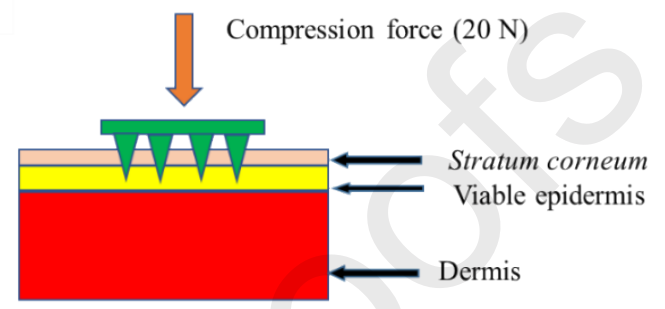

Schematic diagram for HFMN insertion into the skin

G

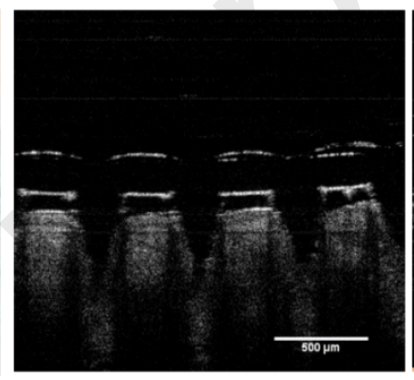

H

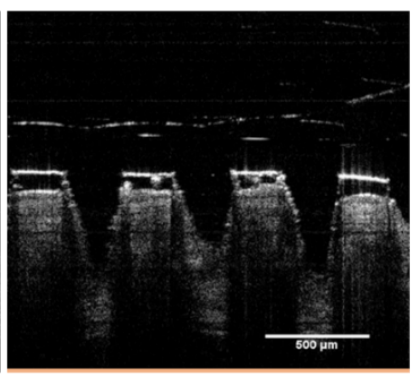

Figure 6. Representative samples of microscopic images for HFMN-F8 (A); HFMN-F10 (B), the black scale bar in both images A and B represents a length of $1 \mathrm{~mm}$; Percentage of MN height reduction and a sample of light microscopic images for compressed MN from both HFMN-F8 and HFMN-F10 after applying a compression force of 32 N/Array $(\mathbf{C})$; a schematic diagram showing the experimental set up for the process of insertion of HFMN into an excised full-thickness neonatal porcine skin model (D); Light microscopic images of skin surface showing the holes created by HFMN-F8 (E); HFMN-F10 (F) following staining with methylene blue aqueous solution, the black scale bar in both images $E$ and $F$ represents a length of $2 \mathrm{~mm}$; representative samples of optical coherence tomography (OCT) image showing the insertion depth of HFMN-F8 (G) and HFMN-F10 (H) in excised fullthickness neonatal porcine skin, the white scale bar in both images $\mathrm{G}$ and $\mathrm{H}$ represents a length of $0.5 \mathrm{~mm}$.

HFMN-F8 exhibited satisfactory mechanical properties, which were demonstrated by a \%MNs` height reduction of approximately $14.73 \pm 1.91 \%$. However, HFMN-F10 showed a significantly $(p<0.05)$ better mechanical strength where the $\%$ height reduction was of the magnitude $10.22 \pm 2.58 \%$. It is worth mentioning that the fabricated HFMN became slightly flexible when stored under ambient room conditions due to moisture absorption. To avoid this problem, they were stored either in a moisture-impermeable aluminium bag or in a desiccator on silica gel under vacuum at the ambient room temperature. Additionally, we observed that 
the stored HFMN were physically stable, and we were able to use them even after three months from storage.

With respect of insertion properties, both HFMN-F8 and HFMN-F10 were able to pierce the outermost layer of the excised full-thickness neonatal porcine skin when applied by thumb (Figure 6(E and F)). The insertion was confirmed by visualising the pores created by the MN after treating the skin with methylene blue dye that selectively marks sites of skin penetration. Methylene blue is highly water-soluble, producing an intense blue-coloured solution. Thus, when the dye is applied to the microporated skin, it preferentially diffuses into the aqueous microchannels that take up the stain, whereas the rest of the skin remains impermeable to the dye [26,34]. The OCT images also confirmed that both HFMN were inserted well into the skin (Figure $6(\mathrm{G}$ and $\mathrm{H})$ ). Measuring the height of part of the needle that went beneath the skin surface revealed that the mean insertion depths for HFMN-F8 and HFMN-F10 were $491.6 \pm 18.2$ and $498.9 \pm 10.9 \mu \mathrm{m}$, respectively. These equate to 67.7 and $68.7 \%$ of the MN height, respectively. Taking into consideration that the neonatal porcine skin is a useful model for human skin [39,63], we can conclude that our HFMN, both HFMN-F8 and HFMN-F10 would be able to pierce the outermost layer of the human skin.

It observed, even though our HFMN were mechanically robust, they did not insert fully into the skin. This can be explained by the deformation of the skin's surface known to occur during MN insertion due to the skin`s viscoelasticity [26,64]. The relatively broad base (300 $\mu \mathrm{m})$ and the conical shape of the needle contributed to this incomplete insertion. From a clinical point of view, complete insertion is not necessary, and a partial microneedle insertion is sufficient for the HFMN to reach the interstitial fluid and swell to form microconduits, allowing drug diffusion from its attached patch-like drug reservoir to the dermal microcirculation $[25,34]$.

\subsection{Preparation and characterisation of $M T X-R V$}

The aim here was to develop MTX-RV, which will be combined with the HFMN and allow delivery of MTX in a sustained manner. Three MTX-RVs were prepared and characterised. The favoured MTX-RV was that demonstrated the following characteristics: Chemically compatible with MTX, high drug loading, uniform appearance, robust for ease of handling and shows slow dissolution in PBS ( $\mathrm{pH}$ 7.4). The latter property may suggest that MTX-RV would help in sustaining MTX delivery through the swollen HFMN following their 
insertion into the skin. All MTX-RV were homogenous, robust, and no drug aggregates or precipitation were observed. The characteristics of the three MTX-RV are outlined in Table 5.

Table 5. The characteristics of MTX-RVs prepared from various polymeric blends. Data reported as means $\pm \mathrm{SD}$, $\mathrm{n}=3$.

\begin{tabular}{cccc}
\hline Characteristics & MTX-RV1 & MTX-RV2 & MTX-RV3 \\
\hline Dried weight $(\mathrm{mg})$ & $3880 \pm 350$ & $2270 \pm 220$ & $1550 \pm 170$ \\
Surface area $\left(\mathrm{cm}^{2}\right)$ & 30 & 30 & 30 \\
Thickness $\left(\mathrm{mm}^{2}\right)$ & $0.94 \pm 0.14$ & $0.54 \pm 0.18$ & $0.42 \pm 0.10$ \\
Density $\left(\mathrm{g} / \mathrm{cm}^{3}\right)$ & $1.20 \pm 0.13$ & $1.4 \pm 0.15$ & $1.23 \pm 0.13$ \\
Moisture content $(\% \mathrm{w} / \mathrm{w})$ & $18.8 \pm 2.2$ & $8 \pm 2.3$ & $14.5 \pm 2.9$ \\
Drug content $(\mu \mathrm{g} / \mathrm{mg})$ & $66.9 \pm 3.7$ & $119.7 \pm 0.7$ & $150.3 \pm 5.3$ \\
Dissolution time $(\mathrm{min})$ & $43.0 \pm 6.0$ & $53.1 \pm 7.0$ & $73.3 \pm 6.1$ \\
\hline
\end{tabular}

MTX-RV3 (prepared from HPMC with added glycerol) had shown to have the most desirable properties, as it was homogenous, and no drug precipitation or aggregation was noticed. Also, the drug was stable as no degradation was seen even after storing in a moisture impermeable aluminium bag for 1-month (data is not reported). Also, MTX-RV3 had the highest MTX loading (150.3 $\mu \mathrm{g} / \mathrm{mg})$ and the longest dissolution time (73.3 min). Therefore, it was selected as the finalised MTX-RV and used in subsequent studies. It is important to note that adding glycerol was essential for all polymeric blends, as it helped in forming resilient MTX-RV.

\subsection{Ex vivo permeation studies}

The finalised HFMN, namely HFMN-F8 and HFMN-F10, were combined with the finalised MTX-RV3, to form integrated patch-F8 and integrated patch-F10, respectively. These patches were tested for delivering MTX (ex vivo) using an excised dermatomed porcine skin and compared with a control (MTX permeation from MTX-RV3 by passive diffusion). MTX permeation profiles are presented in Figure 7. As can be seen, applying MTX-RV3 directly to the dermatomed neonatal porcine skin without HFMN assistance (control), resulted in only a small quantity of MTX being delivered, which was approximately $9.9 \pm 3.2 \mu \mathrm{g} / \mathrm{cm}^{2}$ over $24 \mathrm{~h}$. This equates to $0.11 \pm 0.3 \%$ of the applied dose $\left(8.5 \pm 0.9 \mathrm{mg} / \mathrm{cm}^{2}\right)$. The steady-state flux of MTX was $0.4 \pm 0.1 \mu \mathrm{g} . \mathrm{h} / \mathrm{cm}^{2}$. In contrast, both integrated patches dramatically $(p<0.0001)$ enhanced MTX delivery compared to the control set-up. For example, the integrated patch-F8 delivered MTX at a steady-state flux of $603.5 \pm 119.3 \mu \mathrm{g} . \mathrm{h} / \mathrm{cm}^{2}$, which was 1508 -fold higher than that achieved with the control. It was observed that the integrated patch-F10 delivered 
MTX at a slower steady-state flux of $506.8 \pm 136.9 \mu \mathrm{g} . \mathrm{h} / \mathrm{cm}^{2}$ in comparison with that achieved with the integrated patch-F8, suggesting that it could possibly exert better control on MTX permeation rate. However, statistically, there was no significant difference $(p>0.95)$ between the two fluxes.

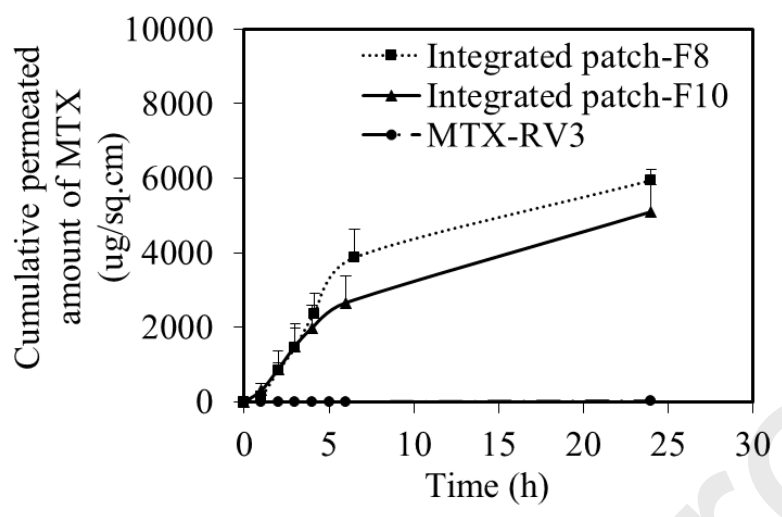

Figure 7. Ex vivo permeation profiles of MTX from its methotrexate reservoir (MTX-RV3) by passive diffusion and from the integrated patch-F8 and the integrated patch-F10. Data is reported as means $\pm S D, n=3$.

The amount of MTX delivered over $24 \mathrm{~h}$ from the integrated patch-F8, and integrated patch-F10 were $5930.6 \pm 290.6$ and $5083 \pm 742.6 \mu \mathrm{g} / \mathrm{cm}^{2}$, respectively. These equate to means of 69.7 and $69.5 \%$ of the applied doses $\left(8.5 \pm 0.6\right.$ and $6.8 \pm 0.4 \mathrm{mg} / \mathrm{cm}^{2}$, respectively). The results suggest that both integrated patches can deliver MTX efficiently in a sustained manner. However, HFMN-F10 exhibited better control, which is more desirable. The differences in MTX steady-state fluxes between the ex vivo study using HFMN and the in vitro permeation study using HFFs, which was reported in Section 3.1.4, is probably due to the fact, that in the in vitro permeation study, fully hydrated HFFs were used. Thus the effective diffusional area did not change over the experiment time course. However, in the ex vivo permeation study, dry HFMN were used and, upon insertion, they swelled up to $340.6 \%$ for HFMN-F10 and $255.9 \%$ for HFMN-F8, which possibly led to a substantial increase in the diffusional surface area. Based on so far generated data, it could be concluded that both patches satisfy the essential properties in terms of mechanical strength, swelling and robustness. However, the integrated patch-F10 was chosen for the subsequent in vivo study because it exhibited better mechanical strength and provided slightly better control of MTX permeation rate, which could help in delivering MTX in a sustained manner.

Several studies have employed MN to deliver MTX transdermally, Vemulapalli et al. [65] have shown that they could deliver MTX transdermally at a mean rate of $18.2 \mathrm{ug} . \mathrm{cm}^{2} / \mathrm{h}$ using dissolving maltose-based MN by "poke and patch" approach combined with iontophoresis. 
Also, Du et al. [21] have used a dissolving hyaluronic acid-based MN to deliver MTX intradermally, and the delivered dose was limited to approximately $13.8 \mu \mathrm{g} / 10 \mathrm{x} 10 \mathrm{MN}$ array, which could be useful for psoriasis treatment but not for treatment of RA and JIA. Interestingly, this study showed that our novel drug delivery system was able to deliver a substantial percentage $(69.5 \%)$ of the applied dose $\left(6.8 \pm 0.4 \mathrm{mg} / \mathrm{cm}^{2}\right)$ of MTX at significantly $(p<0.0001)$ higher steady-state flux of $506.8 \pm 136.9 \mu \mathrm{g} . \mathrm{h} / \mathrm{cm}^{2}$ and in a sustained manner over $24 \mathrm{~h}$, demonstrating its superiority in comparison with any other reported transdermal delivery system for MTX and, thus supporting its potential use to benefit patients.

\subsection{In vivo studies}

To demonstrate the utility and advantages of our novel drug delivery system, we carried out an in vivo study using Sprague Dawley (SD) rats. In this study, MTX is used. Thus, measuring its concentrations in the blood would help in evaluating its pharmacokinetics behaviour following its administration by our novel drug delivery system in comparison with the conventional oral administration route $[14,66]$. However, this will be of little value in evaluating MTX therapeutic efficacy. This is because MTX efficacy is not correlated with only MTX concentrations in the blood, but with the total concentration of its metabolites, which are produced by glutamation the red blood cells (RBCs) [67]. To elaborate more on this, following MTX administration, its concentrations in the serum falls rapidly as it is transported into the RBCs. Once inside, MTX undergo a polyglutamatation process, and up to 5 glutamate moieties are added via enzyme folylpolyglutamate synthetase. As a group, these products of intracellular polyglutamation are referred to as $\mathrm{MTX}-\mathrm{PG}_{\mathrm{n}}$, where $\mathrm{n}$ represents the number of glutamate moieties [67]. The parent drug of MTX in the RBCs will have one glutamate moiety and, therefore, is known as MTX glutamate $1\left(\mathrm{MTX}-\mathrm{PG}_{1}\right)$. Numerous studies have suggested a strong correlation between the concentrations of the long-chained $\mathrm{MTX}_{\mathrm{P}} \mathrm{PG}_{3-5}$ in the RBCs, in particular $\mathrm{MTX}_{-} \mathrm{PG}_{3}$, and controlling the disease activity [67-69]. Therefore, in this study, all MTX- $\mathrm{PG}_{1-5}$ were quantified to evaluate the effect of our novel drug delivery system and the administration route on MTX polyglutamatation process in the RBCs and the MTX-PGs pharmacokinetic profiles in comparison with the conventional oral administration route (as a control). 


\subsubsection{HPLC-MS-MS validation}

In the in vivo experiment, MTX and its polyglutamates $\left(\mathrm{MTX}-\mathrm{PG}_{1-5}\right)$ were quantified using the validated HPLC-MS-MS method as per ICH standard. The run time was 20 min, with all peaks of interest being eluted within $18 \mathrm{~min}$. No interference with any impurities from their biological matrix was observed. The detector response was linear in the range of $0.25-100$ $\mu \mathrm{g} / \mathrm{ml}\left(\mathrm{R}^{2}>0.995\right)$ for all five individual $\mathrm{MTX}-\mathrm{PG}_{1-5}$. Precision and accuracy were found to

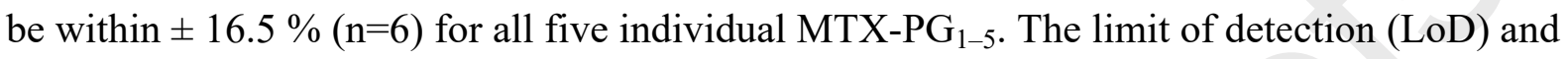
the limit of quantitation (LoQ) were 1.2 and $3.5 \mathrm{nM}$ for all five individual $\mathrm{MTX}-\mathrm{PG}_{1-5}$.

\subsubsection{MTX and its polyglutamates pharmacokinetics}

MTX and its polyglutamates (MTX- $\left.\mathrm{PG}_{1-5}\right)$ pharmacokinetic profiles following MTX oral and transdermal administration are presented in Figure 8. MTX-PG1 pharmacokinetics were calculated and outlined in Table 6. As can be seen in Figure 8A, in the oral group, MTX$\mathrm{PG}_{1}$ appeared in the blood rapidly and reached its maximum concentration $\left(\mathrm{C}_{\max }\right)$ of $57.4 \pm$ $20.0 \mathrm{nM}$ at $1 \mathrm{~h}$ (the first sampling point), and then quickly declined to reach $15.4 \pm 7.5 \mathrm{nM}$ at $2 \mathrm{~h}$ and then increased again to reach $41.1 \pm 16.1 \mathrm{nM}$ at $3 \mathrm{~h}$. After that, MTX-PG1 concentration decreased to $10.5 \pm 2.7 \mathrm{nM}$ at $6 \mathrm{~h}$ and continued decreasing gradually to reach its lowest concentration of $3.6 \pm 1.2 \mathrm{nM}$ at the end of the study period $(48 \mathrm{~h})$. The $\mathrm{AUC}_{0-48}$ was $485.9 \pm$ 2.7 nM.h. In contrast, in the transdermal group (Figure 8B), the integrated-patch-F10 produced a progressive increase in MTX-PG1 blood concentrations over $24 \mathrm{~h}$. At $1 \mathrm{~h}$, the concentration was $7.6 \pm 2.0 \mathrm{nM}$, and it then increased gradually to reach its peak concentration $\left(\mathrm{C}_{\max }=\right.$ was $35.1 \pm 5.1 \mathrm{nM})$ at $24 \mathrm{~h}$. After that, the concentration had fallen steadily to $3.75 \pm 0.35 \mathrm{nM}$ at 48 h. The $\mathrm{AUC}_{0-48}$ was found to be $685.9 \pm 157.9$ nM.h, which was around 1.2-fold higher than the $\mathrm{AUC}_{0-48}(485.9 \pm 127.9$ nM.h) observed in the oral group. 

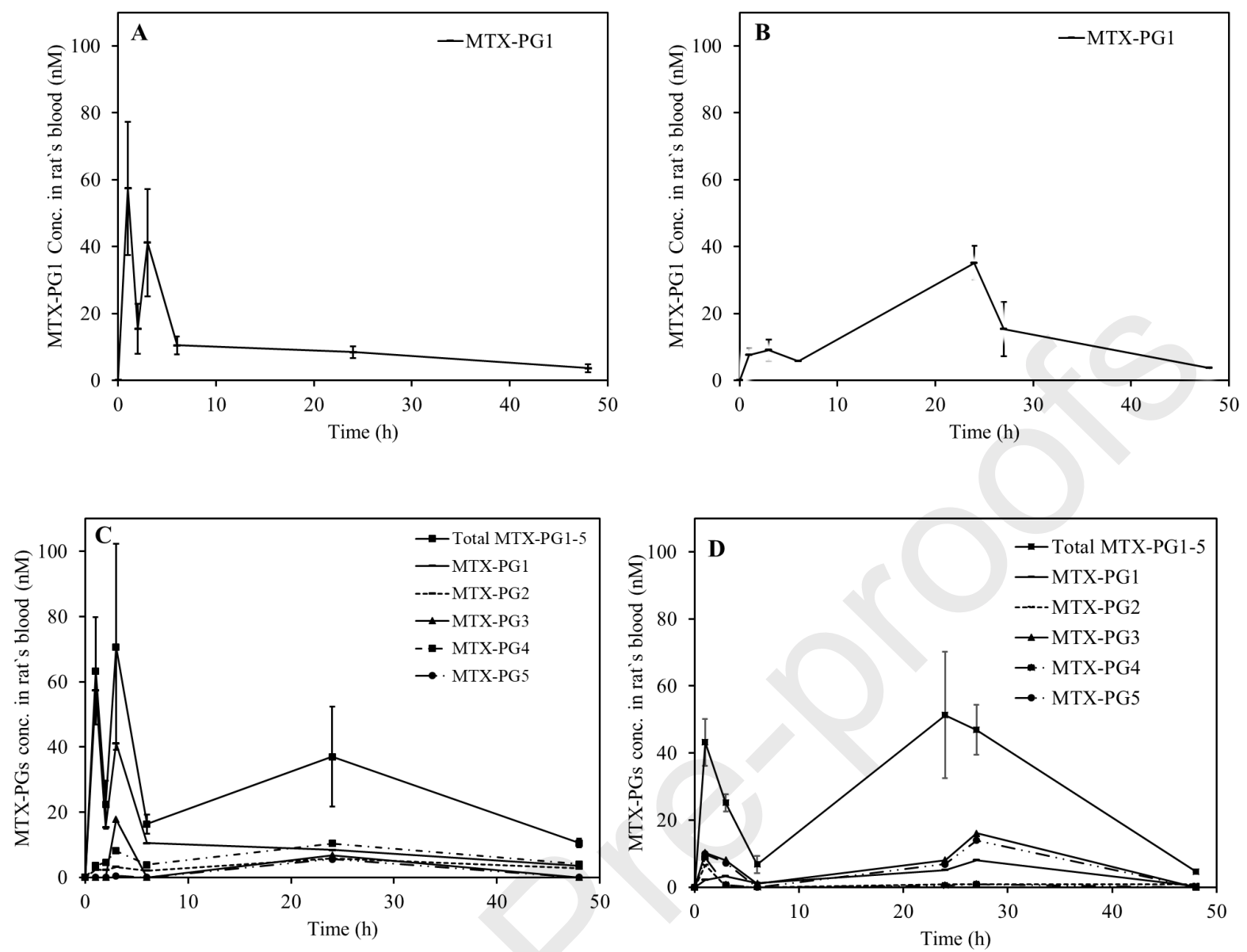

Figure 8. Pharmacokinetic profile of MTX-PG1 following MTX administration orally using MTX aqueous solution (A); Pharmacokinetic profile of MTX-PG1 following MTX administration transdermally using the integrated patch-F10 (B); Pharmacokinetic profile of $\mathrm{MTX}_{-} \mathrm{PG}_{1-5}$ following MTX administration orally using MTX aqueous (C) and Pharmacokinetic profile of $\mathrm{MTX}-\mathrm{PG}_{1-5}$ following MTX administration transdermally using the integrated patch-F10 (D). Data is reported as means $\pm \mathrm{SD}, \mathrm{n} \geq 3$.

As can be seen from Table $6, \mathrm{C}_{\max }$ in the transdermal group was significantly $(p<0.05)$ lower than $\mathrm{C}_{\max }$ in the oral group. Indeed, it is approximately $60 \%$ of $\mathrm{C}_{\max }$ in the oral group. However, the $\mathrm{AUC}_{0-48}$ in the transdermal group was 1.2-fold higher than the $\mathrm{AUC}_{0-48}$ in the oral group. It was indicating that the integrated patch-F10 delivered MTX in a sustained manner and avoided the high blood peak concentration $\left(\mathrm{C}_{\max }\right)$, which was observed in the oral group, without affecting the systemic exposure (which is related to the AUC). These findings are consistent with the results obtained from the in vitro and ex vivo studies reported in the previous sections and give further support to our hypothesis that our novel drug delivery would be able to deliver MTX transdermally in a sustained manner over $24 \mathrm{~h}$, with significantly lower $\mathrm{C}_{\max }$, while maintaining the same or even better-delivered dose than that achieved by the conventional oral administration route. 
This is very important from a clinical point of view, as several studies demonstrated that avoiding the high peak concentrations of MTX in blood by splitting the weekly dose into smaller doses helped improve MTX efficacy and reduce the side effects [14,66,70]. This suggests that our drug delivery system could be used as an alternative minimally invasive drug delivery system to deliver MTX efficiently in a sustained manner, (where $C_{\max }$ was significantly lower than that obtained from orally administered MTX, even though the delivered dose was higher), which could possibly help in reducing the side effects associate with oral and subcutaneous administration.

Table 6. A summary of the MTX pharmacokinetic parameters in rats following its administration either transdermally by as an "integrated patch"-F10 or orally using MTX aqueous solution. Data is reported as means $\pm \mathrm{SD}, \mathrm{n} \geq 3$.

\begin{tabular}{ccc}
\hline $\begin{array}{c}\text { Pharmacokinetic } \\
\text { parameters }\end{array}$ & $\begin{array}{c}\text { MTX aqueous } \\
\text { solution } \\
\text { (Oral administration) }\end{array}$ & $\begin{array}{c}\text { MTX in the integrated patch-F10 } \\
\text { (Transdermal delivery) }\end{array}$ \\
\hline Dose $(\mathrm{mg} / \mathrm{kg})$ & $2.66 \pm 0.05$ & $5.23 \pm 0.54$ \\
$\mathrm{~T} \max (\mathrm{h})$ & 1 & 24 \\
$\mathrm{C} \max (\mathrm{nM})$ & $57.4 \pm 20.0$ & $35.1 \pm 5.1$ \\
$\mathrm{AUC}_{0-48}(\mathrm{nM} . \mathrm{h})$ & $485.9 \pm 127.9$ & $685.3 \pm 157.9$ \\
$\mathrm{AUC}_{0-\mathrm{INF}}(\mathrm{nM} . \mathrm{h})$ & $626.2 \pm 147.5$ & $730.2 \pm 176.3$ \\
\hline
\end{tabular}

With respect to the effect of our drug delivery system and the new administration route on MTX polyglutamation process, $\left(\mathrm{MTX}-\mathrm{PG}_{1-5}\right)$ pharmacokinetics in the blood are presented in Figure $8(\mathrm{~B}$ and $\mathrm{C})$. As can be seen, all $\mathrm{MTX}^{-} \mathrm{PG}_{1-5}$ appeared in blood in both groups at various concentrations. Importantly, the long-chain $\mathrm{MTX}-\mathrm{PG}_{3-5}$ were the predominant metabolite in both groups, in particular $\mathrm{MTX}-\mathrm{PG}_{3}$, which is 10 -fold more potent than MTX itself in controlling RA and JIA [67,69]. $\mathrm{C}_{\max }$ of the total $\mathrm{MTX}-\mathrm{PG}_{1-5}$, in the oral and transdermal groups, was $63.3 \pm 16.5$ and $51.4 \pm 18.9 \mathrm{nM}$, respectively, indicating that our drug delivery system and the transdermal route did not significantly alter MTX polyglutamation process and the long-chained $\mathrm{MTX}-\mathrm{PG}_{3-5}$ are still the primary MTX metabolites. Therefore, delivering MTX by our integrated patch would possibly provide the same control for RA and JIA (comparable to that obtained by MTX administered by oral route).

In terms of the safety of our novel drug delivery system, as can be seen in Figure 9A, the HFMN-F10 in the dry state appeared intact and none of the MN were shattered or broken. When they were removed from the rats' skin following application for $24 \mathrm{~h}$, the HFMN-F10 were noticeably swollen, where the length of each $\mathrm{MN}$ was nearly doubled, but they were 
removed macroscopically intact from the skin, with no individual $\mathrm{MN}$ lost or tips missing (Figure 9B). Moreover, from a safety standpoint, it is also imperative that our drug delivery system does not cause any irritation, either due to MTX, which is a cytotoxic drug, or the HFMN themselves, at the application site. The created microholes should close soon after HFMN removal to prevent permeation of undesired substances or pathogenic microorganisms [71]. Therefore, the skin condition was closely monitored for $24 \mathrm{~h}$ following HFMN removal. Images of the skin at the HFMN application site are shown in Figure $9(\mathrm{C}-\mathrm{F})$. Microscopic holes in the skin (Figure 9C), which were created by the HFMN, were macroscopically closed within 30 min (Figure 9D) following HFMN removal and could barely be identified by eye after $2 \mathrm{~h}$ (Figure 9E). This can be ascribed to the skin's natural repair mechanism whereby, upon disruption of the stratum corneum barrier, lamellar body secretion is immediately initiated, followed by synthesis of lipids to restore and maintain the barrier [72,73].

Furthermore, although MTX is a cytotoxic drug and the HFMN was applied for $24 \mathrm{~h}$, no irritation was observed at the application site, apart from mild erythema (Figure 9C), which had faded within $2 \mathrm{~h}$ (Figure 9D) and continued to resolve over time, so that it could hardly be noticed after $24 \mathrm{~h}$ (Figure 9F). This result is in line with other studies that found erythema caused by MNs insertion is transient and disappears over a short period [28,74,75]. This can possibly be ascribed to the fact that our HFMN were prepared from biocompatible materials (PVP, PVA and CA), which have long histories of safe use, as we have mentioned in the previous section (3.1). Additionally, the MTX did not induce any skin irritation, or localised cytotoxic effects. This may be because MTX did not accumulate to toxic levels at the application site, which is related to the sustained release of MTX from its patch-like reservoir and its high aqueous solubility [20,76] facilitating its fast clearance from the application site by the skin microcirculation. Our HFMN themselves were not sterilised and the skin at the application site was not even cleansed before their application. Despite these HFMN puncturing the skin's protective stratum corneum barrier, no signs of infection (redness, swelling, pus, oozing or weeping) were noticed either immediately after HFMN removal (Figure 9C) or even after $24 \mathrm{~h}$ (Figure 9F). Our findings here are very much in line with other studies, which reported that MNs do not to cause infection [27,28,77,78]. This was expected and can be ascribed to the skin's natural repair and defensive mechanisms [75,77]. Taken all together, our novel drug delivery system may be considered safe to use. However, further studies will, in due course, be required to evaluate the system's performance and the impact of this on the skin when repeatedly applied, as envisaged for clinical treatment. 
Despite the convenience of applying such a drug delivery system for $24 \mathrm{~h}$, currently, no MNbased patches are available on the market. However, it has been shown that applying MNbased patches once, as well as multiple times, is well-tolerated and did not induce any problems $[75,79]$.

A

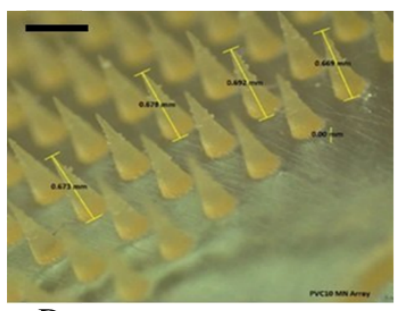

$\mathrm{C}$

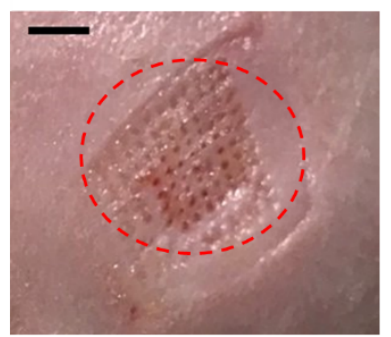

$\mathrm{D}$

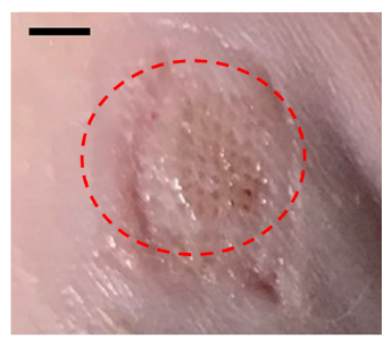

B

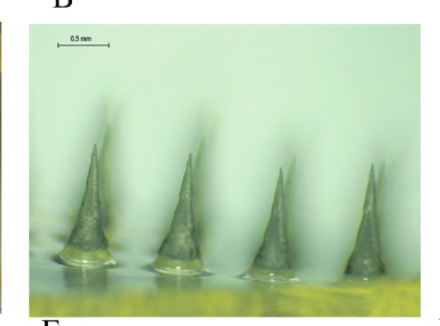

E

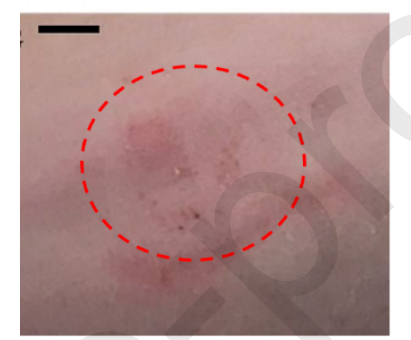

$\mathrm{F}$

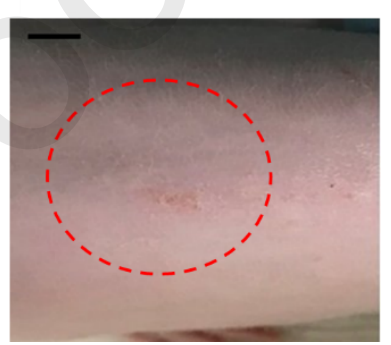

Figure 9. Microscopic images of HFMN-F10 before insertion into skin, the black scale bar represents a length of $0.5 \mathrm{~mm}(\mathbf{A})$; swollen intact HFMN-F10 after removing from rats skin following $24 \mathrm{~h}$ application, the black scale bar represents a length of $0.5 \mathrm{~mm}(\mathbf{B})$; digital images showing the micro holes created by HFMN in rats skin immediately after HFMN removal and that no irritation at the application site but only mild erythema (C), the microholes visibly closed after 30 min from removing the HFMN (D) the microholes cannot be identified visibly after $2 \mathrm{~h}$ from removing the HFMN (E); no sign of infection (redness, swelling, pus, oozing or weeping) at the application site even after 24 from removing the $\operatorname{HFMN}(\mathbf{F})$.

Although our novel drug delivery system demonstrated superior properties and capability in delivering MTX, it showed some limitations, including the lower \%SE in comparison with other HFMN such as Gantrez ${ }^{\circledR}$ AN-139-based HFMN and that \%GF did not reach $100 \%$. Regarding the $\% \mathrm{SE}$, our HFMN-F10 exhibited \%SE equals to $340.6 \pm 14.9 \%$ which represents approximately $31.7 \%$ of that reported for Gantrez ${ }^{\circledR}$ AN-139-based HFMN, which was $1071 \pm 106 \%$ [26]. However, the lower $\%$ SE reduced the rate of MTX delivery but not the extent. In fact, this was proven to be an advantage for us, as we were aiming to deliver MTX in a sustained manner. In terms of \%GF, our studies showed that there is a small proportion of the polymer still unreacted, which is mostly PVP, and it may undesirably leach into the skin. However, this should not be an issue, since HFMN have a relatively small weight (approximately $20 \mathrm{mg} / 0.5 \mathrm{~cm}^{2}$ array). Thus, the amount of the polymer that might leach from the HFMN into the skin would be potentially around $2 \mathrm{mg} / 0.5 \mathrm{~cm}^{2}$ array $(10.4 \%$ of the HFMN weight) at maximum. Furthermore, PVP is a biocompatible polymer and is widely used in fabricating dissolving MNs and other drug delivery systems [80-82]. It is also used as a plasma 
expander [34]. It has relatively low molecular weight (MW $<60,000 \mathrm{Da}$ ), facilitating it excretion from the body [70].

This study presents a proof-of-concept for the feasibility of using the HFMN as minimally invasive drug delivery system to deliver MTX in a sustained manner, which avoids the GIT tract and possibly could minimise the side effects, mainly nausea and vomiting, associated with the conventional administration routes.

Although to take this new drug delivery system forward, further studies are needed, we can use the above-generated data to cautiously estimate the size of the integrated patch that is required to deliver one of the most common doses of MTX in treatment of RA and JIA in humans, i.e. $25 \mathrm{mg}$ once weekly [3,12]. This approach is widely used for dose scaling between humans and animals [83,84]. Taking into consideration that, (i) MTX administered at a dose of $2.5 \mathrm{mg} / \mathrm{kg}$ to rats produces a comparable area-under-the-curve (AUC) to the AUC in humans after giving the same treatment $(2.5 \mathrm{mg} /$ person) orally [42], (ii) in our in vivo study, the integrated patch $\left(1 \mathrm{~cm}^{2}\right.$ in total size, with drug loading $\left.5.23 \pm 0.54 \mathrm{mg}\right)$ produced an $\mathrm{AUC}_{0-48 \mathrm{~h}}$ equal to 1.2-fold of the $\mathrm{AUC}_{0-48 \mathrm{~h}}$ obtained from orally administered MTX dose $(2.66 \pm 0.05$ $\mathrm{mg} / \mathrm{kg}$ ) in the Sprague Dawley rats. Hence, the patch size, which would be able to produce AUC equivalent to the AUC, which may be obtained from administering the most common therapeutic dose in humans (i.e. $25 \mathrm{mg}=10 \times 2.5 \mathrm{mg})$ will be in the magnitude of $(1 / 1.2 \times 10$ $\left.=8.33 \mathrm{~cm}^{2} \approx 9 \mathrm{~cm}^{2}\right)$.

Currently, no MN patch is available in the market. Nevertheless, we would expect such a patch size is still very feasible based on the study which has been done in our group [79]. We have shown that a patch comprised of 16 individual $0.5 \mathrm{~cm}^{2} \mathrm{MN}$ arrays (equal to $8 \mathrm{~cm}^{2}$ patch size) was applied and successfully inserted manually by human volunteers with minimal simple instruction. Although $\mathrm{MN}$ patch is different from the currently marked transdermal patches, the concept of such a relatively big patch size is generally accepted by the patients and it is well within the range of marketed transdermal patches. Indeed, Novartis markets Nicotinell ${ }^{\circledR}$ (nicotine) patches of $30 \mathrm{~cm}^{2}$, while Janssen markets Duragesic ${ }^{\circledR}$ CII (fentanyl) patches of 32 and $42 \mathrm{~cm}^{2}$.

\section{Conclusions}

The current work reports a successful development of novel hydrogel-forming microneedles arrays (HFMN), prepared from PVA/PVP blend crosslinked with CA by heating 
and combined with a patch-like reservoir loaded with MTX (MTX-RV) forming an integrated patch. The integrated patch was able to bypass the skin barrier and deliver MTX in a sustained manner over $24 \mathrm{~h}$. Importantly, the HFMN were removed intact from the skin with only mild erythema, despite the cytotoxic nature of MTX. Accordingly, the integrated patch produced in this work represents a promising minimally invasive transdermal drug delivery system that can overcome the skin barrier and deliver MTX in a sustained manner. This may help in minimising or even avoiding nausea and vomiting, associated with the conventional administration routes.

It is worth noting that this drug delivery system is not limited to MTX delivery. Still, it may also be used to deliver other drugs, thus, greatly expanding the range of medicines that can be delivered transdermally to the benefit of patients and industry.

Before this novel drug delivery system can reach clinical practice and achieve patient benefits, other studies should be carried out to evaluate its efficiency, usability, and acceptability to inform and ensure its maximum impact. Currently, we are working on assessing the safety and effectiveness of this drug delivery system in delivering MTX in multiple application scenarios and investigating its usefulness to deliver other drug candidates.

\section{Acknowledgements}

This work was generously supported by Versus Arthritis, UK (20938) and in part by the Wellcome Trust (WT094085MA). The contents are the responsibility Queen's University Belfast and do not necessarily reflect the views of Versus Arthritis, UK. Thanks to Stephen Lloyd for his help in drug dosing to the animals in the in vivo study.

\section{Conflict of Interest}

The authors have no conflict of interest to declare.

\section{References}

[1] G. Ferrara, G. Mastrangelo, P. Barone, F. La Torre, S. Martino, G. Pappagallo, A. Ravelli, A. Taddio, F. Zulian, R. Cimaz, Rheumatology Italian Study Group, Methotrexate in juvenile idiopathic arthritis: advice and recommendations from the MARAJIA expert consensus meeting, Pediatr. Rheumatol. 16 (2018) 46. https://doi.org/10.1186/s12969-018-0255-8. 
[2] C.G. Shinde, M.P. Venkatesh, T.M.P. Kumar, H.G. Shivakumar, Methotrexate: A Gold Standard for Treatment of Rheumatoid Arthritis, J. Pain Palliat. Care Pharmacother. 28 (2014) 351-358. https://doi.org/10.3109/15360288.2014.959238.

[3] A. Klein, I. Kaul, I. Foeldvari, G. Ganser, A. Urban, G. Horneff, Efficacy and safety of oral and parenteral methotrexate therapy in children with juvenile idiopathic arthritis: An observational study with patients from the German Methotrexate Registry, Arthritis Care Res. (Hoboken). 64 (2012) 1349-1356. https://doi.org/10.1002/acr.21697.

[4] P.C. Taylor, A. Balsa Criado, A.-B. Mongey, J. Avouac, H. Marotte, R.B. Mueller, How to Get the Most from Methotrexate (MTX) Treatment for Your Rheumatoid Arthritis Patient?-MTX in the Treat-to-Target Strategy, J. Clin. Med. 8 (2019) 515. https://doi.org/10.3390/jcm8040515.

[5] G.A. Vena, N. Cassano, F. Iannone, Update on subcutaneous methotrexate for inflammatory arthritis and psoriasis., Ther. Clin. Risk Manag. 14 (2018) 105-116. https://doi.org/10.2147/TCRM.S154745.

[6] A.E. Bello, E.L. Perkins, R. Jay, P. Efthimiou, Recommendations for optimizing methotrexate treatment for patients with rheumatoid arthritis, (2017). https://doi.org/10.2147/OARRR.S131668.

[7] J.C. Branco, A. Barcelos, F.P. de Araújo, G. Sequeira, I. Cunha, J.V. Patto, M. Oliveira, M.P. Mateus, M. Couto, P. Nero, P. Pinto, P. Monteiro, W. Castelão, J. Félix, D. Ferreira, J. Almeida, M.J. Silva, Utilization of Subcutaneous Methotrexate in Rheumatoid Arthritis Patients After Failure or Intolerance to Oral Methotrexate: A Multicenter Cohort Study, Adv. Ther. 33 (2016) 46-57. https://doi.org/10.1007/s12325-015-0276-3.

[8] J. Jacobse, W. ten Voorde, R. Rissmann, J. Burggraaf, R. ten Cate, L. Schrier, The effect of repeated methotrexate injections on the quality of life of children with rheumatic diseases, Eur. J. Pediatr. 178 (2019) 17-20. https://doi.org/10.1007/s00431018-3286-8.

[9] J. Braun, P. Kästner, P. Flaxenberg, J. Währisch, P. Hanke, W. Demary, U. von Hinüber, K. Rockwitz, W. Heitz, U. Pichlmeier, C. Guimbal-Schmolck, A. Brandt, Comparison of the clinical efficacy and safety of subcutaneous versus oral administration of methotrexate in patients with active rheumatoid arthritis: Results of a six-month, multicenter, randomized, double-blind, controlled, phase IV trial, Arthritis 
Rheum. 58 (2008) 73-81. https://doi.org/10.1002/art.23144.

[10] J. Braun, R. Rau, An update on methotrexate, Curr. Opin. Rheumatol. 21 (2009) 216223. https://doi.org/10.1097/BOR.0b013e328329c79d.

[11] M.K. Rohr, T.R. Mikuls, S.B. Cohen, J.C. Thorne, J.R. O’Dell, Underuse of Methotrexate in the Treatment of Rheumatoid Arthritis: A National Analysis of Prescribing Practices in the US, Arthritis Care Res. 69 (2017) 794-800. https://doi.org/10.1002/acr.23152.

[12] K. Visser, D. van der Heijde, Optimal dosage and route of administration of methotrexate in rheumatoid arthritis: a systematic review of the literature., Ann. Rheum. Dis. 68 (2009) 1094-9. https://doi.org/10.1136/ard.2008.092668.

[13] B. Shea, M. V. Swinden, E.T. Ghogomu, Z. Ortiz, W. Katchamart, T. Rader, C. Bombardier, G.A. Wells, P. Tugwell, Folic acid and folinic acid for reducing side effects in patients receiving methotrexate for rheumatoid arthritis, J. Rheumatol. 41 (2014) 1049-1060. https://doi.org/10.3899/jrheum.130738.

[14] M. Hoekstra, C. Haagsma, C. Neef, J. Proost, A. Knuif, M. van de Laar, Splitting highdose oral methotrexate improves bioavailability: a pharmacokinetic study in patients with rheumatoid arthritis., J. Rheumatol. 33 (2006) 481-5. http://www.ncbi.nlm.nih.gov/pubmed/16463431 (accessed December 18, 2019).

[15] W. Wang, H. Zhou, L. Liu, Side effects of methotrexate therapy for rheumatoid arthritis: A systematic review, Eur. J. Med. Chem. 158 (2018) 502-516. https://doi.org/10.1016/j.ejmech.2018.09.027.

[16] M.H. Schiff, J.S. Jaffe, B. Freundlich, Head-to-head, randomised, crossover study of oral versus subcutaneous methotrexate in patients with rheumatoid arthritis: drugexposure limitations of oral methotrexate at doses $\geq 15 \mathrm{mg}$ may be overcome with subcutaneous administration, Ann. Rheum. Dis. 73 (2014) 1549-1551. https://doi.org/10.1136/annrheumdis-2014-205228.

[17] M.A. Bechard, J.R. Lemieux, J. Roth, K.W. Duffy, C.M. Duffy, M.O. Aglipay, R. Jurencak, Procedural pain and patient-reported side effects with weekly injections of subcutaneous Methotrexate in children with rheumatic disorders, Pediatr. Rheumatol. Online J. 12 (2014). https://doi.org/10.1186/1546-0096-12-54.

[18] R. Prasad, V. Koul, Transdermal delivery of methotrexate: past, present and future 
prospects, Ther. Deliv. 3 (2012) 315-325. https://doi.org/10.4155/tde.12.3.

[19] A. Williams, Transdermal and topical drug delivery from theory to clinical practice, Pharmaceutical Press, 2003.

[20] A.M. dos Santos, F.C. Carvalho, D.A. Teixeira, D.L. Azevedo, W.M. de Barros, M.P.D. Gremião, Computational and experimental approaches for development of methotrexate nanosuspensions by bottom-up nanoprecipitation, Int. J. Pharm. 524 (2017) 330-338. https://doi.org/10.1016/j.ijpharm.2017.03.068.

[21] H. Du, P. Liu, J. Zhu, J. Lan, Y. Li, L. Zhang, J. Zhu, J. Tao, Hyaluronic Acid-Based Dissolving Microneedle Patch Loaded with Methotrexate for Improved Treatment of Psoriasis, ACS Appl. Mater. Interfaces. (2019). https://doi.org/10.1021/acsami.9b15668.

[22] M.J. Abla, A. Chaturvedula, C. O’Mahony, A.K. Banga, Transdermal delivery of methotrexate for pediatrics using silicon microneedles, Ther. Deliv. 4 (2013) 543-551. https://doi.org/10.4155/tde.13.24.

[23] H.X. Nguyen, A.K. Banga, Delivery of Methotrexate and Characterization of Skin Treated by Fabricated PLGA Microneedles and Fractional Ablative Laser, Pharm. Res. 35 (2018). https://doi.org/10.1007/s11095-018-2369-6.

[24] K. Ita, Transdermal Delivery of Drugs with Microneedles-Potential and Challenges, Pharmaceutics. 7 (2015) 90-105. https://doi.org/10.3390/pharmaceutics7030090.

[25] R.F. Donnelly, T.R.R. Singh, M.J. Garland, K. Migalska, R. Majithiya, C.M. McCrudden, P.L. Kole, T.M.T. Mahmood, H.O. McCarthy, A.D. Woolfson, Hydrogelforming microneedle arrays for enhanced transdermal drug delivery, Adv. Funct. Mater. 22 (2012) 4879-4890. https://doi.org/10.1002/adfm.201200864.

[26] R.F. Donnelly, M.T.C. McCrudden, A. Zaid Alkilani, E. Larrañeta, E. McAlister, A.J. Courtenay, M.-C. Kearney, T.R.R. Singh, H.O. McCarthy, V.L. Kett, E. CaffarelSalvador, S. Al-Zahrani, A.D. Woolfson, Hydrogel-forming microneedles prepared from "super swelling" polymers combined with lyophilised wafers for transdermal drug delivery., PLoS One. 9 (2014) e111547. https://doi.org/10.1371/journal.pone.0111547.

[27] A.J. Courtenay, M.T.C. McCrudden, K.J. McAvoy, H.O. McCarthy, R.F. Donnelly, Microneedle-mediated transdermal delivery of bevacizumab, Mol. Pharm. 15 (2018) 
3545-3556. https://doi.org/10.1021/acs.molpharmaceut.8b00544.

[28] E.M. Migdadi, A.J. Courtenay, I.A. Tekko, M.T.C. McCrudden, M.C. Kearney, E. McAlister, H.O. McCarthy, R.F. Donnelly, Hydrogel-forming microneedles enhance transdermal delivery of metformin hydrochloride, J. Control. Release. 285 (2018) 142151. https://doi.org/10.1016/j.jconrel.2018.07.009.

[29] M. Ventre, V. Coppola, M. Iannone, P.A. Netti, I. Tekko, E. Larrañeta, A.M. Rodgers, C.J. Scott, A. Kissenpfennig, R.F. Donnelly, S. Maher, D. Losic, A. George, A. Ramachandran, Nanotechnologies for tissue engineering and regeneration, Nanotechnologies Prev. Regen. Med. (2018) 93-206. https://doi.org/10.1016/B978-0323-48063-5.00002-2.

[30] E. Larrañeta, M. Henry, N.J. Irwin, J. Trotter, A.A. Perminova, R.F. Donnelly, Synthesis and characterization of hyaluronic acid hydrogels crosslinked using a solvent-free process for potential biomedical applications, Carbohydr. Polym. 181 (2018) 1194-1205. https://doi.org/10.1016/j.carbpol.2017.12.015.

[31] T.R. Raj Singh, P.A. McCarron, A.D. Woolfson, R.F. Donnelly, Investigation of swelling and network parameters of poly(ethylene glycol)-crosslinked poly(methyl vinyl ether-co-maleic acid) hydrogels, Eur. Polym. J. 45 (2009) 1239-1249. https://doi.org/10.1016/j.eurpolymj.2008.12.019.

[32] C. Birck, S. Degoutin, N. Tabary, V. Miri, M. Bacquet, New crosslinked cast films based on poly(vinyl alcohol): Preparation and physico-chemical properties, Express Polym. Lett. 8 (2014) 941-952. https://doi.org/10.3144/expresspolymlett.2014.95.

[33] R.F. Donnelly, R. Majithiya, T.R.R. Singh, D.I.J. Morrow, M.J. Garland, Y.K. Demir, K. Migalska, E. Ryan, D. Gillen, C.J. Scott, A.D. Woolfson, Design, Optimization and Characterisation of Polymeric Microneedle Arrays Prepared by a Novel Laser-Based Micromoulding Technique, Pharm. Res. 28 (2011) 41-57. https://doi.org/10.1007/s11095-010-0169-8.

[34] E. Larrañeta, R.E.M. Lutton, A.D. Woolfson, R.F. Donnelly, Microneedle arrays as transdermal and intradermal drug delivery systems: Materials science, manufacture and commercial development, Mater. Sci. Eng. R Reports. 104 (2016) 1-32. https://doi.org/10.1016/J.MSER.2016.03.001.

[35] R. Wong, M. Ashton, K. Dodou, Effect of Crosslinking Agent Concentration on the 
Properties of Unmedicated Hydrogels, Pharmaceutics. 7 (2015) 305-319. https://doi.org/10.3390/pharmaceutics7030305.

[36] M.M. M.L. Mansour, T.B. Mahmoud, Synthesis and swelling characterization of cross-linked pvp/pva hydrogels, Iran. Polym. J. 14 (2005) 1022-1030.

[37] T.R. Raj Singh, M.J. Garland, K. Migalska, E.C. Salvador, R. Shaikh, H.O. McCarthy, A. David Woolfson, R.F. Donnelly, Influence of a pore-forming agent on swelling, network parameters, and permeability of poly(ethylene glycol)-crosslinked poly(methyl vinyl ether-co-maleic acid) hydrogels: Application in transdermal delivery systems, J. Appl. Polym. Sci. 125 (2012) 2680-2694. https://doi.org/10.1002/app.36524.

[38] R.F. Donnelly, T.R.R. Singh, M.J. Garland, K. Migalska, R. Majithiya, C.M. McCrudden, P.L. Kole, T.M.T. Mahmood, H.O. McCarthy, A.D. Woolfson, HydrogelForming Microneedle Arrays for Enhanced Transdermal Drug Delivery, Adv. Funct. Mater. 22 (2012) 4879-4890. https://doi.org/10.1002/adfm.201200864.

[39] E. Abd, S.A. Yousef, M.N. Pastore, K. Telaprolu, Y.H. Mohammed, S. Namjoshi, J.E. Grice, M.S. Roberts, Skin models for the testing of transdermal drugs, Clin. Pharmacol. Adv. Appl. 8 (2016) 163-176. https://doi.org/10.2147/CPAA.S64788.

[40] J.B. Nielsen, I. Plasencia, J.A. Sørensen, L.A. Bagatolli, Storage conditions of skin affect tissue structure and subsequent in vitro percutaneous penetration, Skin Pharmacol. Physiol. 24 (2011) 93-102. https://doi.org/10.1159/000322304.

[41] A.M. Barbero, H.F. Frasch, Effect of Frozen Human Epidermis Storage Duration and Cryoprotectant on Barrier Function Using Two Model Compounds, Skin Pharmacol. Physiol. 29 (2016) 31-40. https://doi.org/10.1159/000441038.

[42] T. Kuroda, K. Namba, T. Torimaru, K. Kawashima, M. Hayashi, Species differences in oral bioavailability of methotrexate between rats and monkeys, Biol. Pharm. Bull. 23 (2000) 334-338. https://doi.org/10.1248/bpb.23.334.

[43] A.F. Hawwa, A. AlBawab, M. Rooney, L.R. Wedderburn, M.W. Beresford, J.C. McElnay, A Novel Dried Blood Spot-LCMS Method for the Quantification of Methotrexate Polyglutamates as a Potential Marker for Methotrexate Use in Children, PLoS One. 9 (2014) e89908. https://doi.org/10.1371/journal.pone.0089908.

[44] E. Begas, C. Papandreou, A. Tsakalof, D. Daliani, G. Papatsibas, E. Asprodini, Simple 
and reliable HPLC method for the monitoring of methotrexate in osteosarcoma patients., J. Chromatogr. Sci. 52 (2014) 590-5.

https://doi.org/10.1093/chromsci/bmt081.

[45] Y. Zhang, M. Huo, J. Zhou, S. Xie, PKSolver: An add-in program for pharmacokinetic and pharmacodynamic data analysis in Microsoft Excel, Comput. Methods Programs Biomed. 99 (2010) 306-314. https://doi.org/10.1016/j.cmpb.2010.01.007.

[46] M.L. M., T.B. MAHMOUD, M. MASOUD, SYNTHESIS AND SWELLING CHARACTERIZATION OF CROSS-LINKED PVP/PVA HYDROGELS, 14 (2005) 1022-1030.

[47] M.I. Baker, S.P. Walsh, Z. Schwartz, B.D. Boyan, A review of polyvinyl alcohol and its uses in cartilage and orthopedic applications, J. Biomed. Mater. Res. Part B Appl. Biomater. 100B (2012) 1451-1457. https://doi.org/10.1002/jbm.b.32694.

[48] T. Kamarul, G. Krishnamurithy, N.D. Salih, N.S. Ibrahim, H.R.B. Raghavendran, A.R. Suhaeb, D.S.K. Choon, Biocompatibility and toxicity of poly(vinyl alcohol)/N,Ocarboxymethyl chitosan scaffold., ScientificWorldJournal. 2014 (2014) 905103. https://doi.org/10.1155/2014/905103.

[49] E.-R. Kenawy, M.H. El-Newehy, F.I. Abdel-Hay, A.E.-R.R. El-Shanshoury, Synthesis and biocidal activity of modified poly(vinyl alcohol), Arab. J. Chem. 7 (2014) 355361. https://doi.org/10.1016/J.ARABJC.2013.04.005.

[50] E.A. Kamoun, X. Chen, M.S. Mohy Eldin, E.-R.S. Kenawy, Crosslinked poly(vinyl alcohol) hydrogels for wound dressing applications: A review of remarkably blended polymers, Arab. J. Chem. 8 (2015) 1-14. https://doi.org/10.1016/J.ARABJC.2014.07.005.

[51] S.H. Hyon, W.I. Cha, Y. Ikada, M. Kita, Y. Ogura, Y. Honda, Poly(vinyl alcohol) hydrogels as soft contact lens material., J. Biomater. Sci. Polym. Ed. 5 (1994) 397406.

[52] H.X. Nguyen, B.D. Bozorg, Y. Kim, A. Wieber, G. Birk, D. Lubda, A.K. Banga, Poly (vinyl alcohol) microneedles: Fabrication, characterization, and application for transdermal drug delivery of doxorubicin, Eur. J. Pharm. Biopharm. 129 (2018) 88103. https://doi.org/10.1016/J.EJPB.2018.05.017.

[53] L.K. Vora, P.R. Vavia, E. Larrañeta, S.E.J. Bell, R.F. Donnelly, Novel 
nanosuspension-based dissolving microneedle arrays for transdermal delivery of a hydrophobic drug, J. Interdiscip. Nanomedicine. 3 (2018) 89-101. https://doi.org/10.1002/jin2.41.

[54] H.R. Nejad, A. Sadeqi, G. Kiaee, S. Sonkusale, Low-cost and cleanroom-free fabrication of microneedles, Microsystems Nanoeng. 4 (2018) 17073. https://doi.org/10.1038/micronano.2017.73.

[55] Y.K. Demir, Z. Akan, O. Kerimoglu, Characterization of Polymeric Microneedle Arrays for Transdermal Drug Delivery, PLoS One. 8 (2013) e 77289. https://doi.org/10.1371/journal.pone.0077289.

[56] S. Yang, Y. Feng, L. Zhang, N. Chen, W. Yuan, T. Jin, A scalable fabrication process of polymer microneedles., Int. J. Nanomedicine. 7 (2012) 1415-22. https://doi.org/10.2147/IJN.S28511.

[57] X. Yang, Q. Liu, X. Chen, F. Yu, Z. Zhu, Investigation of PVA/ws-chitosan hydrogels prepared by combined $\gamma$-irradiation and freeze-thawing, Carbohydr. Polym. 73 (2008) 401-408. https://doi.org/10.1016/j.carbpol.2007.12.008.

[58] CFR - Code of Federal Regulations Title 21, (n.d.).

[59] H.M. Zidan, E.M. Abdelrazek, A.M. Abdelghany, A.E. Tarabiah, Characterization and some physical studies of PVA/PVP filled with MWCNTs, J. Mater. Res. Technol. (2018). https://doi.org/10.1016/J.JMRT.2018.04.023.

[60] J. Thomas, K. Gomes, A. Lowman, M. Marcolongo, The effect of dehydration history on PVA/PVP hydrogels for nucleus pulposus replacement, J. Biomed. Mater. Res. 69B (2004) 135-140. https://doi.org/10.1002/jbm.b.20023.

[61] A. Joshi, G. Fussell, J. Thomas, A. Hsuan, A. Lowman, A. Karduna, E. Vresilovic, M. Marcolongo, Functional compressive mechanics of a PVA/PVP nucleus pulposus replacement, Biomaterials. 27 (2006) 176-184. https://doi.org/10.1016/J.BIOMATERIALS.2005.06.003.

[62] E. Larrañeta, L. Barturen, M. Ervine, R.F. Donnelly, Hydrogels based on poly(methyl vinyl ether-co-maleic acid) and Tween 85 for sustained delivery of hydrophobic drugs, Int. J. Pharm. 538 (2018) 147-158. https://doi.org/10.1016/j.ijpharm.2018.01.025.

[63] W. Meyer, [Comments on the suitability of swine skin as a biological model for human skin]., Hautarzt. 47 (1996) 178-82. 
[64] E. Larrañeta, J. Moore, E.M. Vicente-Pérez, P. González-Vázquez, R. Lutton, A.D. Woolfson, R.F. Donnelly, A proposed model membrane and test method for microneedle insertion studies, Int. J. Pharm. 472 (2014) 65-73. https://doi.org/10.1016/j.ijpharm.2014.05.042.

[65] V. Vemulapalli, Y. Yang, P.M. Friden, A.K. Banga, Synergistic effect of iontophoresis and soluble microneedles for transdermal delivery of methotrexate, J. Pharm. Pharmacol. 60 (2008) 27-33. https://doi.org/10.1211/jpp.60.1.0004.

[66] P. Dhaon, S.K. Das, R. Srivastava, G. Agarwal, A. Asthana, Oral Methotrexate in split dose weekly versus oral or parenteral Methotrexate once weekly in Rheumatoid Arthritis: a short-term study, Int. J. Rheum. Dis. 21 (2018) 1010-1017. https://doi.org/10.1111/1756-185X.12910.

[67] T. Dervieux, R. Zablocki, J. Kremer, Red blood cell methotrexate polyglutamates emerge as a function of dosage intensity and route of administration during pulse methotrexate therapy in rheumatoid arthritis, Rheumatology. 49 (2010) 2337-2345. https://doi.org/10.1093/rheumatology/keq216.

[68] P. Angelis-Stoforidis, F.J.E. Vajda, N. Christophidis, Methotrexate polyglutamate levels in circulating erythrocytes and polymorphs correlate with clinical efficacy in rheumatoid arthritis, Clin. Exp. Rheumatol. 17 (1999) 313-320.

[69] J.M. Dalrymple, L.K. Stamp, J.L. O’Donnell, P.T. Chapman, M. Zhang, M.L. Barclay, Pharmacokinetics of oral methotrexate in patients with rheumatoid arthritis, Arthritis Rheum. 58 (2008) 3299-3308. https://doi.org/10.1002/art.24034.

[70] G.D. Weinstein, P. Frost, Methotrexate for psoriasis. A new therapeutic schedule., Arch. Dermatol. 103 (1971) 33-8. http://www.ncbi.nlm.nih.gov/pubmed/5539502 (accessed December 18, 2019).

[71] J. Gupta, H.S. Gill, S.N. Andrews, M.R. Prausnitz, Kinetics of skin resealing after insertion of microneedles in human subjects, J. Control. Release. 154 (2011) 148-155. https://doi.org/10.1016/j.jconrel.2011.05.021.

[72] P.M. Elias, J.S. Wakefield, An integrated view of the epidermal environmental interface, Dermatologica Sin. 33 (2015) 49-57. https://doi.org/10.1016/j.dsi.2015.03.008.

[73] A.G. Harris, C. Naidoo, D.F. Murrell, Skin needling as a treatment for acne scarring: 
An up-to-date review of the literature, Int. J. Women's Dermatology. 1 (2015) 77-81. https://doi.org/10.1016/j.ijwd.2015.03.004.

[74] D.P. Wermeling, S.L. Banks, D.A. Hudson, H.S. Gill, J. Gupta, M.R. Prausnitz, A.L. Stinchcomb, Microneedles permit transdermal delivery of a skin-impermeant medication to humans, Proc. Natl. Acad. Sci. U. S. A. 105 (2008) 2058-2063. https://doi.org/10.1073/pnas.0710355105.

[75] R. Al-Kasasbeh, A.J. Brady, A.J. Courtenay, E. Larrañeta, M.T.C. McCrudden, D. O'Kane, S. Liggett, R.F. Donnelly, Evaluation of the clinical impact of repeat application of hydrogel-forming microneedle array patches, Drug Deliv. Transl. Res. 10 (2020) 690-705. https://doi.org/10.1007/s13346-020-00727-2.

[76] N.A. Kasim, M. Whitehouse, C. Ramachandran, M. Bermejo, H. Lennernäs, A.S. Hussain, H.E. Junginger, S.A. Stavchansky, K.K. Midha, V.P. Shah, G.L. Amidon, Molecular properties of WHO essential drugs and provisional biopharmaceutical classification., Mol. Pharm. 1 (2004) 85-96. https://doi.org/10.1021/mp034006h.

[77] R.F. Donnelly, A.D. Woolfson, Patient safety and beyond: What should we expect from microneedle arrays in the transdermal delivery arena?, Ther. Deliv. 5 (2014) 653-662. https://doi.org/10.4155/tde.14.29.

[78] J.H. Cary, B.S. Li, H.I. Maibach, Dermatotoxicology of microneedles (MNs) in man, Biomed. Microdevices. 21 (2019). https://doi.org/10.1007/s10544-019-0371-3.

[79] A. Ripolin, J. Quinn, E. Larrañeta, E.M. Vicente-Perez, J. Barry, R.F. Donnelly, Successful application of large microneedle patches by human volunteers, Int. J. Pharm. 521 (2017) 92-101. https://doi.org/10.1016/j.ijpharm.2017.02.011.

[80] J. Zhao, Y. Wu, J. Chen, B. Lu, H. Xiong, Z. Tang, Y. Ji, In vivo monitoring of microneedle-based transdermal drug delivery of insulin, J. Innov. Opt. Health Sci. 11 (2018) 1850032. https://doi.org/10.1142/S1793545818500323.

[81] R.R.S. Thakur, I.A. Tekko, F. Al-Shammari, A.A. Ali, H. McCarthy, R.F. Donnelly, Rapidly dissolving polymeric microneedles for minimally invasive intraocular drug delivery, Drug Deliv. Transl. Res. 6 (2016) 800-815. https://doi.org/10.1007/s13346016-0332-9.

[82] M. Teodorescu, M. Bercea, Poly(vinylpyrrolidone) - A versatile polymer for biomedical and beyond medical applications, Polym. - Plast. Technol. Eng. 54 (2015) 
923-943. https://doi.org/10.1080/03602559.2014.979506.

[83] M.T.C. Mc Crudden, E. Larrañeta, A. Clark, C. Jarrahian, A. Rein-Weston, S. LachauDurand, N. Niemeijer, P. Williams, C. Haeck, H.O. McCarthy, D. Zehrung, R.F. Donnelly, Design, formulation and evaluation of novel dissolving microarray patches containing a long-acting rilpivirine nanosuspension, J. Control. Release. 292 (2018) 119-129. https://doi.org/10.1016/j.jconrel.2018.11.002.

[84] A.D. Permana, I.A. Tekko, M.T.C. McCrudden, Q.K. Anjani, D. Ramadon, H.O. McCarthy, R.F. Donnelly, Solid lipid nanoparticle-based dissolving microneedles: A promising intradermal lymph targeting drug delivery system with potential for enhanced treatment of lymphatic filariasis, J. Control. Release. 316 (2019) 34-52. https://doi.org/10.1016/j.jconrel.2019.10.004.

\section{CRediT authorship contribution statement}

Conceptualization: I.A.T. and R.F.D; Methodology: I.A.T, G.C., I.M.N.H. and L.V.; Software: I.A.T.; Validation: I.A.T. and G.C.; Formal analysis: I.A.T and G.C.; Investigation: I.A.T., J.C.M., H.O.M., M.R and R.F.D; Resources: J.C.M., H.O.M., M.R and R.F.D; Data curation: I.A.T and G.C.; Writing — original draft preparation: I.A.T.; Writing—review and editing: I.A.T, J.D.R., R.R.S.T. M.R. and R.F.D; Visualization: I.A.T, J.D. R. and E.L.; Supervision: J.C.M., H.O.M, M.R. and R.F.D; Project administration: I.A.T, J.C.M., M.R. and R.F.D; Funding acquisition: J.C.M., H.O.M., M.R and R.F.D.

\section{Conflict of Interest}

The authors have no conflict of interest to declare.

\section{Highlights}

- Hydrogel-forming microneedle arrays (HFMN) were produced for the first time from an aqueous PVA/PVP blend crosslinked with citric acid by heating.

- The HFMN showed a satisfactory mechanical strength, excellent insertion capability and swelled moderately. 
- A patch-like MTX reservoir (MTX-RV) was prepared from HPMC and glycerol.

- Both MTX-RV and HFMN were combined and formed an integrated patch, which was able to deliver MTX transdermally in a sustained manner over $24 \mathrm{~h}$ in ex vivo and in vivo setup. 